\title{
Newer-generation antihistamines and the risk of adverse events in children: a systematic review
}

\author{
Michael Miligkos ${ }^{1}$, Maria Dakoutrou $^{1}$, Eleni Statha ${ }^{2}$, Nikoletta Theochari ${ }^{2}$, Ilektra Antonia \\ Mavroeidi $^{2}$, oanna Pankozidou ${ }^{2}$, Irene Papaconstadopoulos ${ }^{3}$, and Nikolaos Papadopoulos ${ }^{4}$ \\ ${ }^{1}$ Agia Sofia Children's Hospital \\ ${ }^{2}$ Society of Junior Doctors \\ ${ }^{3}$ Monmouth University \\ ${ }^{4}$ National \& Kapodistrian University of Athens
}

December 1, 2020

\begin{abstract}
Background: H1-antihistamines (AHs) are widely used for the treatment of allergic diseases, being one of the most commonly prescribed classes of medications in Pediatrics. Newer-generation AHs are associated with fewer adverse effects compared to first-generation. However, their relative harms in the pediatric population still need scrutiny. Methods: We performed a systematic review of randomized controlled trials (RCTs) which included comparisons of safety parameters between an orally administered newer-generation AH with another AH (first- or second- generation), montelukast or placebo in children aged[?]12 years. We searched MEDLINE and CENTRAL, independently extracted data on study population, interventions, adverse events (AEs) and treatment discontinuations, and assessed the methodological quality of the included RCTs using the Cochrane's risk of bias tool. Results: Fourty-five RCTs published between 1989 and 2017 met eligibility criteria. The majority of RCTs included school-aged children with allergic rhinitis and had a follow-up period of up to a month. Four RCTs reported serious AEs in patients receiving a newer-generation $\mathrm{AH}$, but only two patients experienced a possibly drug-related serious AE. The occurrence of AEs, drug-related AEs and treatment discontinuations due to AEs varied between RCTs. Most AEs reported were of mild intensity. Indirect evidence indicates that cetirizine is more sedating than the other newer-generation AHs. Conclusion: Our findings confirm that newer-generation AHs have a favorable safety and tolerability profile. However, we could not draw firm conclusions regarding the comparative safety profile of the newer-generation AHs due to the paucity of head-to-head RCTs, variation in definitions and reporting of AEs, and short follow-up duration.
\end{abstract}

\section{Introduction}

Allergic conditions affect a large number of children worldwide and have considerable socioeconomic burdens for both the children and their families ${ }^{1}$. It is estimated that approximately $40 \%$ of children suffer from allergic rhinitis, whereas almost half of the school-age population is sensitized to one or more common allergens, and these numbers are expected to rise following a steady upward trend in the last decades ${ }^{1,2}$.

$\mathrm{H}_{1}$-antihistamines are widely used for the treatment of allergic diseases, being one of the most commonly prescribed classes of medications in the pediatric population ${ }^{3}$. They mainly act in the respiratory, gastrointestinal and vascular smooth muscle tissues by preventing their constriction and in the salivary and lacrimal gland tissues by decreasing the histamine induced secretion ${ }^{4}$. More than 45 antihistamines are currently available $^{5}$ and they are generally subdivided into two groups: first- and newer-generation antihistamines. First-generation antihistamines are still widely used in clinical practice worldwide despite the relative paucity of data regarding their use in children. Newer-generation antihistamines are generally associated with fewer adverse effects compared to first-generation antihistamines due to their higher selectivity for the H1-receptor 
and their limited blood-brain barrier passage and therefore are the preferred medications for the treatment of allergic diseases ${ }^{6,7}$. In adults, cetirizine and levocetirizine seem to be more sedating than loratadine, desloratadine and fexofenadine ${ }^{8}$, whereas in a review of inter-drug differences using proportional impairment ratios, minor differences were observed in the likely impairing potential of newer-generation antihistamines ${ }^{9}$. In children, some newer-generation antihistamines are approved from the age of 6 months and most of them from the age of 2 years ${ }^{10}$. However, the relative harms of the newer-generation antihistamines have not been established in the pediatric population.

Therefore, we did a systematic review of randomized controlled trials (RCTs) that compared the safety of newer antihistamines with any other antihistamine, montelukast or placebo in children [?]12 years old.

\section{Methods}

\section{Data sources and search}

We searched MEDLINE and CENTRAL from inception through September 2020. Our search strategy included terms suggestive of the intervention of interest (newer-generation antihistamines) and the age group of interest (pediatric patients [?] 12 years old). Details of our search strategy can be found in the Appendix.

\section{Study selection}

We included peer-reviewed publications of RCTs if they fulfilled all of the following criteria: comparison of a newer-generation antihistamine (bilastine, cetirizine, desloratadine, fexofenadine, levocetirizine, loratadine and rupatadine) with another antihistamine (first- or newer- generation), except for terfenadine/astemizole, montelukast or placebo in children aged [?] 12 years; oral administration of the interventions in any dose and for at least one week (single-dose and provocation challenge studies were excluded in order to better reflect everyday clinical practice); reporting of adverse events; and English-language publication.. The primary outcome of interest was the number of patients with at least one adverse event. Secondary outcomes of interest comprised the type of adverse events, adverse events related to study medication according to the original investigators, serious adverse events and treatment discontinuations due to adverse events. Two investigators (M.D. and E.S.) independently screened the titles and abstracts of the citations for potentially relevant publications using Abstrackr ${ }^{11}$. The same two investigators retrieved and screened the full-text of potentially relevant articles. Any disagreement was resolved with the assistance of a third investigator (M.M.).

\section{Data extraction}

Two investigators (M.D. and E.S.) independently extracted data from the included publications. Any disagreement was resolved by a third investigator (M.M.). We extracted data on study design and methods, patient characteristics, interventions, comparators, concomitant medications, adverse events and treatment discontinuations using a standardized extraction form in an Excel@ spreadsheet.

\section{Quality assessment}

We assessed the methodological quality of the included publications using the Cochrane's Collaboration risk of bias tool for $\mathrm{RCTs}^{12}$. We assigned a judgement of 'low', 'high' or 'unclear' to every item included in the tool (sequence generation, allocation concealment, patients' blinding, caregivers' blinding, outcome assessors' blinding, attrition, and selective outcome reporting). Studies that reported sufficient information about the implementation of blinding (such as double-blinding and matching capsules) were considered low risk of bias for this specific item, whereas studies that reported only phrases such as "double-blind" had unclear risk of bias. In double-blind studies, the blinding of the outcome assessors was considered adequate if patients reported the occurrence of adverse events. We compared the proportion of patients who discontinued treatment in each group by the chi-square test and a p-value less than 0.1 was indicative of differential lossto-follow-up and the study was assigned a judgement of 'high risk of bias' for this specific item. Two of three authors (N.T., I.M. and J.P.) independently completed the assessments. Any disagreements were resolved by the addition of a third author (M.M.). 


\section{Results}

\section{Literature search}

Figure 1 summarizes our search yield. We screened 2358 citations, we excluded 2249 as irrelevant, and 109 articles were retrieved for full-text review. Finally, we included 45 randomized controlled trials ${ }^{13-59}$. There were two RCTs with data reported in two separate publications each ${ }^{43 \& 44,48 \& 49}$.

\section{Study characteristics}

Table 1 summarizes the characteristics of the included studies that were published between 1989 - 2017 .

The majority of studies were conducted in Europe and USA. The RCTs included children 6 months to 15 years old with an overall male predominance. Most studies included school-aged children and seven publications included children [?]24 months-old ${ }^{20,25,27,31,35,43 \& 44}$. The most frequent type of condition that required the use of antihistamines was allergic rhinitis ${ }^{13,14,15,17-21,23,24,26,28-30,32-36,39-42,45-52,55-58}$, followed by chronic idiopathic urticaria ${ }^{15,20,30,35,38,42}$ and atopic dermatitis ${ }^{25,35,37,43,44,54}$. The majority of studies did not provide any evidence regarding concomitant medications and ten studies reported inclusion of patients with concomitant asthma ${ }^{16,17,28,33,34,40,47,52-54}$. Cetirizine was administered in 22 studies ${ }^{14,21,26,32,34-36,38,40,45,47-58}$, loratadine in 10 studies $^{14,18,31,37,39,42,46-47,59}$, levocetirizine in 6 studies $^{19-21,25,28,29}$, fexofenadine in 4 studies $^{23,24,33,41}$, desloratadine in 4 studies $^{13,16,27,30}$, rupatadine in 2 studies $^{16,17}$ and bilastine in 1 study ${ }^{15}$. In most studies the dose of cetirizine was $0.2 \mathrm{mg} / \mathrm{kg}$ twice daily for children less than 2 years old, 2.5 to 5 mg once daily (OD) for children 2-6 years old and $10 \mathrm{mg}$ OD for children 6-12 years old. Loratadine dosing was generally weight-based in the included studies (5 mg OD in children [?] 30kg and $10 \mathrm{mg}$ OD in children $>30 \mathrm{~kg}$ ). Levocetirizine dose was $5 \mathrm{mg}$ OD for children 6-12 years old, $1.25 \mathrm{mg}$ twice daily (BD) for children 1-5 years old, $0.125 \mathrm{mg} / \mathrm{kg}$ OD for children 1-2 years old and $1.25 \mathrm{mg}$ OD for children 6-11 months old. The dose of fexofenadine ranged from $15 \mathrm{mg}$ to $60 \mathrm{mg}$ OD, based on age. Desloratadine was administered at a dose of $1 \mathrm{mg}$ OD in infants, $1.25 \mathrm{mg}$ OD in children 1-5 years old and $2.5 \mathrm{mg}$ OD in children 6-11 years old. Rupatadine dosing was weight-based, i.e., $2.5 \mathrm{mg}$ OD for children 10-25 kg and $5 \mathrm{mg}$ OD for children > $25 \mathrm{~kg}$. Bilastine dose was $10 \mathrm{mg}$ OD for children 2-12 years old. The duration of treatment varied among studies (1-72 weeks). The majority of studies lasted less than a month.

\section{Assessment of risk of bias}

Table 2 summarizes our risk of bias assessment. Generation of a randomized sequence and allocation concealment were not clearly reported in most RCTs. All but two RCTs reported double-blinding. The other RCTs were open-label ${ }^{18}$ and investigator-blinded ${ }^{59}$. One RCT had differential loss to follow-up ${ }^{24}$. One crossover RCT had a washout period of 7 days ${ }^{34}$.

\section{Adverse events and tolerability}

The proportions of patients with adverse events in each RCT are shown in Table 3. Four RCTs did not provide numerical data regarding adverse events ${ }^{13,22,48 \& 49,53}$.

\section{Serious adverse events}

Thirteen RCTs provided data regarding the occurrence of serious adverse events ${ }^{14,15,19,21,25-29,32,33,36,43 \& 44}$. Four RCTs reported serious adverse events in patients receiving a newer-generation antihistamine $\mathrm{e}^{15,25,33,43 \& 44}$, but only two patients experienced a possibly drug-related serious adverse event. The first patient received fexofenadine (30mg BD) for 14 days and he experienced mild asymptomatic transient neutropenia, which could also be associated with subclinical infections, according to study authors $^{33}$. The second patient, a 14-month-old toddler, had slightly elevated baseline transaminases levels and 5 weeks after the initiation of cetirizine, the investigators observed a 10 -fold increase (AST and ALT $>1000 \mathrm{UI} / \mathrm{ml})^{43 \& 44}$. The toddler remained asymptomatic throughout the study period and the transaminases levels returned to normal a month after treatment discontinuation.

\section{Newer-generation antihistamines in head-to-head RCTs}


Only five RCTs directly compared newer-generation antihistamines ${ }^{14,17,22,45,47}$. In a large $\mathrm{RCT}^{14}$, the (drugrelated) AEs rates were similar in cetirizine and loratadine groups. However, more patients who received loratadine discontinued treatment due to AEs. Cetirizine and loratadine were also compared in a small $\mathrm{RCT}^{47}$; no patient experienced a drug-related AE or discontinued treatment. Patients on rupatadine experienced fewer (drug-related) AEs compared to patients on desloratadine; no data were reported regarding treatment discontinuations ${ }^{16}$.

\section{Newer-generation antihistamines vs. placebo}

Across all placebo-controlled trials, the proportions of patients with AEs varied. Cetirizine was compared with placebo in $16 \mathrm{RCTs}^{14,21,26,32,34-36,40,43 \& 44,48 \& 49,50,53,54,56-58}$. Overall, the occurrence of adverse events was generally similar in both groups. Half of the RCTs provided information regarding drug-related adverse events $^{14,26,35,40,43 \& 44,54,56,57}$. Drug-related AEs occurred in similar frequencies in treatment groups and in less than $5 \%$ of the included patients in RCTs with a large sample size ${ }^{14,43 \& 44}$. The tolerability of cetirizine was comparable to placebo and in eight RCTs no patient discontinued treatment due to AEs in both treatment groups. Loratadine was compared with placebo in seven $\mathrm{RCTs}^{14,22,31,37,39,42,46}$. There were no clinically significant differences between groups in the proportion of patients with (drug-related) AEs or treatment discontinuations due to AEs. Levocetirizine and placebo were compared in six RCTs ${ }^{19,21,23,25,28,29}$. The proportions of patients with (drug-related) AEs or treatment discontinuations were similar between groups. Fexofenadine was compared with placebo in four studies ${ }^{23,24,33,41}$. None of them reported any clinically significant difference regarding AEs or treatment discontinuations. Three studies compared the use of desloratadine with placebo ${ }^{16,27,30}$ and reported slightly lower rates of adverse events in the placebo group. In the largest $\mathrm{RCT}^{27}$, more patients in the desloratadine group experienced drug-related AEs, but less than $1 \%$ of patients discontinued treatment. Rupatadine was compared with placebo in two studies ${ }^{16,17}$. In both studies, the proportions of patients with AEs were similar, however drug-related AEs occurred less frequently in the rupatadine group in one study ${ }^{16}$. No data were available regarding treatment discontinuations. In one RCT, there was no difference between bilastine and placebo in the frequency of AEs, drug-related AEs or discontinuations ${ }^{15}$.

\section{Newer-generation antihistamines vs. first generation antihistamines}

Seven studies compared a newer with a first generation antihistamine, i.e., oxatomide, ketotifen, chlorphenamine, dexchlorphenamine and cyproheptadine $18,22,36,38,51,52,59$. Across all RCTs, the proportions of patients with AEs were slightly lower in the newer-generation antihistamines group compared to the first generation antihistamines group. Only a few patients discontinued treatment in both groups.

\section{Newer-generation antihistamines vs. other drugs}

Cetirizine was compared with montelukast in two small $\mathrm{RCTs}^{26,32}$. No clinically meaningful differences regarding the occurrence of AEs were reported. No patient discontinued treatment in both RCTs.

\section{Newer-generation antihistamines in children [?] 2 years old}

Seven RCTs ${ }^{20,23,25,27,31,35,43 \& 44}$ included infants ([?]6 months old) or young toddlers. There was no difference in the proportion of patients with AEs between the active treatment and placebo groups (Table 3). However, infants who received levocetirizine were more likely to experience an $\mathrm{AE}$ compared to children aged 1-5 years ${ }^{20}$ and cetirizine-related AEs were generally more frequent in the 6-8 month- than the 9-11 month- age group ${ }^{35}$.

\section{Commonly reported adverse events}

Adverse events reported in the included RCTs are summarized in Table 4. The most frequently reported AEs included minor neurological, gastrointestinal and respiratory symptoms and specifically somnolence, headache, insomnia, abdominal pain, vomiting, diarrhea and upper respiratory tract infections. Of note, somnolence was more frequently reported by patients in the cetirizine compared with patients in the placebo groups, whereas no difference was observed between patients treated with cetirizine and first-generation antihistamines. In contrast, the other newer-generation antihistamines did not appear more sedating than 
placebo. In four head-to-head RCTs of cetirizine with loratadine or levocetirizine, the proportions of patients with somnolence were slightly higher in the cetirizine groups, but overall the observed proportions in these RCTs were low ${ }^{14,21,45,47}$. There were no significant differences regarding EEG parameters between any treatment groups in the included RCTs.

\section{Discussion}

In children [?] 12 years old who received newer-generation antihistamines, the occurrence of AEs, drugrelated AEs and treatment discontinuations due to AEs varied between RCTs, though in general, no clinically meaningful differences were observed in the majority of RCTs. Importantly, only two patients experienced a possibly drug-related serious $\mathrm{AE}$ and most AEs reported in the RCTs were of mild intensity.

Several systematic reviews have examined the state of evidence regarding the use of antihistamines, but most of them included adults and/or antihistamines withdrawn from the market. International guidelines such as those published by the European Academy of Allergy and Clinical Immunology (EAACI), the Allergic Rhinitis and its Impact on Asthma (ARIA) group and the Global Allergy and Asthma European Network $\left(\mathrm{GA}^{2} \mathrm{LEN}\right)$ recommend the use of newer-generation over first generation antihistamines in an attempt to avoid sedation and performance impairment associated with the latter type of antihistamines ${ }^{60-62}$. Carson and colleagues ${ }^{8}$ assessed the efficacy and harms of newer-generation antihistamines in head-to-head, placebocontrolled or non-interventional trials in adults and children with allergic rhinitis or urticaria. They concluded that newer-generation antihistamines were well tolerated in children, with no clinically relevant differences regarding adverse events. However, the included studies in adults suggested that newer-generation antihistamines resulted in more sedation compared to placebo and both cetirizine and levocetirizine were more sedating than loratadine or desloratadine. In our study, due to the paucity of head-to-head RCTs, there is only indirect evidence that cetirizine is more sedating than the other newer-generation antihistamines; cetirizine-treated patients experienced somnolence more frequently than placebo-treated patients, whereas no such difference was observed between the remaining newer-generation antihistamines and placebo. In addition, although comparable proportions of patients aged [?] 2 years treated with either a newer-generation antihistamine or placebo reported the occurrence of AEs, these AEs were more frequently reported in the younger age group.

Our systematic review has several limitations, which need to be considered when interpreting the results. At the individual trial level, most RCTs were not primarily designed to assess safety parameters and therefore no formal statistical comparisons were undertaken. The majority of RCTs had a short follow-up period and used patient-reported AEs, through either interviews at trial visits or diary cards. A definition of serious adverse events and data on concomitant medications were generally not reported. Of note, the absence of a sufficient number of head-to-head RCTs provides only indirect evidence for the relative safety and tolerability of newer-generation antihistamines. Although the inclusion of RCTs only in the present systematic review provides the highest level of the available evidence, the exclusion of non-interventional studies which may better reflect everyday clinical practice in certain circumstances and have a longer follow-up period, limit our ability to increase the generalizability of our findings (e.g., reports of off-label use, up-dosing schemes or long-term administration). On the other hand, the exclusion of single-dose RCTs, which objectively measure the effect of antihistamines on alertness and psychomotor performance may have restricted our ability to detect differences of clinical interest. Finally, the observed clinical and methodological heterogeneity (e.g., variation in definitions and monitoring of AEs, administration of antihistamines in patients with different diseases, limited use of validated tools) precluded the quantitative synthesis of the data.

This systematic review investigates the use of newer-generation antihistamines in children. Our findings suggest that these medications have a favorable safety and tolerability profile.. However, we could not draw firm conclusions regarding the comparative safety profile of the newer-generation antihistamines due to the paucity of head-to-head RCTs. Of clinical importance in pediatrics, other factors that may not affect adults should be taken into consideration in the selection of the desired antihistamine, such as taste preference, daily number of doses, volume of dose and safety in cases of overdosing. For example, cetirizine, fexofenadine and levocetirizine should be administered twice daily for optimum concentration in plasma, which may affect 
adherence compared to once daily dosing (10). Well-designed head-to-head RCTs of sufficient duration and with use of validated instruments to assess safety parameters important to patients may help with counselling parents on appropriate use, frequency, dosing and possible adverse effects of newer-generation antihistamines.

(1) Pawankar R, Holgate ST, Canonica GW, Lockey RF, Blaiss MS. WAO White Book on Allergy 2013 Update. World Allergy Organization (WAO); 2013.

(2) Yanai K, Rogala B, Chugh K, Paraskakis E, Pampura AN, Boev R. Safety considerations in the management of allergic diseases: focus on antihistamines. Curr Med Res Opin 2012;28(4):623-642.

(3) Del Cuvillo A, Sastre J, Montoro J, Jauregui I, Ferrer M, Davila I, et al. Use of antihistamines in pediatrics. J Investig Allergol Clin Immunol 2007;17 Suppl 2:28-40.

(4) Fitzsimons R, van der Poel LA, Thornhill W, du Toit G, Shah N, Brough HA. Antihistamine use in children. Arch Dis Child Educ Pract Ed 2015;100(3):122-131.

(5) Simons FE, Simons KJ. Histamine and H1-antihistamines: celebrating a century of progress. J Allergy Clin Immunol2011;128(6):1139-1150.e4.

(6) Church MK, Maurer M, Simons FE, Bindslev-Jensen C, van Cauwenberge P, Bousquet J, et al. Risk of first-generation H(1)-antihistamines: a GA(2)LEN position paper. Allergy 2010;65(4):459-466.

(7) Shimamura T, Shiroishi M, Weyand S, Tsujimoto H, Winter G, Katritch V, et al. Structure of the human histamine H1 receptor complex with doxepin. Nature 2011;475(7354):65-70.

(8) Carson S, Lee N, Thakurta S. Drug Class Review: Newer Antihistamines: Final Report Update 2 [Internet] 2010.

(9) McDonald K, Trick L, Boyle J. Sedation and antihistamines: an update. Review of inter-drug differences using proportional impairment ratios. Hum Psychopharmacol 2008;23(7):555-570.

(10) Parisi GF, Leonardi S, Ciprandi G, Corsico A, Licari A, Miraglia Del Giudice M, et al. Antihistamines in children and adolescents: A practical update. Allergol Immunopathol (Madr) . 2020;S0301-0546(20)30066-5.

(11) Wallace BC, Small K, Brodley CE, Lau J, Trikalinos TA. Deploying an interactive machine learning system in an evidence-based practice center: abstrackr. Proc of the ACM International Health Informatics Symposium (IHI) 2012:819-824.

(12) Higgins JPT, Altman DG, Gotzsche PC, Juni P, Moher D, Oxman AD, et al. The Cochrane Collaboration's tool for assessing risk of bias in randomised trials. BMJ 2011;343:d5928.

(13) Wandalsen GF, Miranda C, Ensina LF, Sano F, Amazonas RB, Silva JMD, et al. Association between desloratadine and prednisolone in the treatment of children with acute symptoms of allergic rhinitis: a double-blind, randomized and controlled clinical trial. Braz J Otorhinolaryngol 2017;83(6):633-639.

(14) Nayak AS, Berger WE, LaForce CF, Urdaneta ER, Patel MK, Franklin KB, et al. Randomized, placebocontrolled study of cetirizine and loratadine in children with seasonal allergic rhinitis. Allergy Asthma Proc 2017;38(3):222-230.

(15) Novak Z, Yanez A, Kiss I, Kuna P, Tortajada-Girbes M, Valiente R. Safety and tolerability of bilastine $10 \mathrm{mg}$ administered for 12 weeks in children with allergic diseases. Pediatr Allergy Immunol 2016; 27(5):493498.

(16) Potter P, Mitha E, Barkai L, Mezei G, Santamaria E, Izquierdo I, et al. Rupatadine is effective in the treatment of chronic spontaneous urticaria in children aged 2-11 years. Pediatr Allergy Immunol2016;27(1):55-61.

(17) Potter P, Maspero JF, Vermeulen J, Barkai L, Nemeth I, Baillieau RA, et al. Rupatadine oral solution in children with persistent allergic rhinitis: A randomized, double-blind, placebo-controlled study.Pediatr Allergy Immunol 2013;24(2):144-150. 
(18) Wu KG, Li TH, Wang TY, Hsu CL, Chen CJ. A comparative study of loratadine syrup and cyproheptadine HCL solution for treating perennial allergic rhinitis in Taiwanese children aged 2-12 years. Int $J$ Immunopathol Pharmacol 2012;25(1):231-237.

(19) Marcucci F, Sensi LG, Abate P, Allocca G, Ugolini E, Di Cara G, et al. Anti-inflammatory activity and clinical efficacy of a 3-month levocetirizine therapy in mite-allergic children. Inflamm Allergy Drug Targets 2011;10(1):32-38.

(20) Hampel F, Ratner P, Haeusler JM. Safety and tolerability of levocetirizine dihydrochloride in infants and children with allergic rhinitis or chronic urticaria. Allergy Asthma Proc2010;31(4):290-295.

(21) Lee CF, Sun HL, Lu KH, Ku MS, Lue KH. The comparison of cetirizine, levocetirizine and placebo for the treatment of childhood perennial allergic rhinitis. Pediatr Allergy Immunol 2009;20(5):493-499.

(22) Ngamphaiboon J, Wirawarn T, Thongkaew T. Prevention of recurrent wheezing in young children by loratadine compared with ketotifen.J Med Assoc Thai 2009;92(3):351-355.

(23) Hampel FC, Kittner B, van Bavel JH. Safety and tolerability of fexofenadine hydrochloride, 15 and 30 mg, twice daily in children aged 6 months to 2 years with allergic rhinitis. Ann Allergy Asthma Immunol 2007;99(6):549-554.

(24) Milgrom H, Kittner B, Lanier R, Hampel FC. Safety and tolerability of fexofenadine for the treatment of allergic rhinitis in children 2 to 5 years old. Ann Allergy Asthma Immunol 2007;99(4):358-363.

(25) Simons FE, Early Prevention of Asthma in Atopic Children (EPAAC) Study Group. Safety of levocetirizine treatment in young atopic children: An 18-month study. Pediatr Allergy Immunol2007;18(6):535-542.

(26) Chen ST, Lu KH, Sun HL, Chang WT, Lue KH, Chou MC. Randomized placebo-controlled trial comparing montelukast and cetirizine for treating perennial allergic rhinitis in children aged 2-6 yr.Pediatr Allergy Immunol 2006;17(1):49-54.

(27) Prenner B, Ballona R, Bueso A, Cardona R, Kim K, Larsen L, et al. Safety of Desloratadine Syrup in Children Six Months to Younger Than 2 Years of Age: A Randomized, Double-Blinded, Placebo-Controlled Study.Pediatric Asthma, Allergy \& Immunology 2006;19(2):91-99.

(28) de Blic J, Wahn U, Billard E, Alt R, Pujazon MC. Levocetirizine in children: evidenced efficacy and safety in a 6-week randomized seasonal allergic rhinitis trial. Pediatr Allergy Immunol2005;16(3):267-275.

(29) Potter PC, Paediatric Levocetirizine Study Group. Efficacy and safety of levocetirizine on symptoms and health-related quality of life of children with perennial allergic rhinitis: a double-blind, placebo-controlled randomized clinical trial. Ann Allergy Asthma Immunol 2005;95(2):175-180.

(30) Bloom M, Staudinger H, Herron J. Safety of desloratadine syrup in children. Curr Med Res Opin 2004;20(12):1959-1965.

(31) Grimfeld A, Holgate ST, Canonica GW, Bonini S, Borres MP, Adam D, et al. Prophylactic management of children at risk for recurrent upper respiratory infections: the Preventia I Study. Clin Exp Allergy 2004;34(11):1665-1672.

(32) Hsieh J, Lue K, Lai D, Sun H, Lin Y. A Comparison of Cetirizine and Montelukast for Treating Childhood Perennial Allergic Rhinitis.Pediatric Asthma, Allergy 85 Immunology 2004;17(1):59-69.

(33) Wahn U, Meltzer EO, Finn AF,Jr, Kowalski ML, Decosta P, Hedlin G, et al. Fexofenadine is efficacious and safe in children (aged 6-11 years) with seasonal allergic rhinitis. J Allergy Clin Immunol2003;111(4):763769.

(34) Segal AT, Meltzer EO, Lockey RF, Prenner BM, Mitchell DQ, Tinkelman DG, et al. Once-Daily Cetirizine Is Safe and Effective for Children with Allergic Rhinitis with and without Intermittent Asthma. Pediatric Asthma, Allergy \&3 Immunology 2003;16(4):265-274. 
(35) Simons FE, Silas P, Portnoy JM, Catuogno J, Chapman D, Olufade AO, et al. Safety of cetirizine in infants 6 to 11 months of age: a randomized, double-blind, placebo-controlled study. J Allergy Clin Immunol 2003;111(6):1244-1248.

(36) Lai DS, Lue KH, Hsieh JC, Lin KL, Lee HS. The comparison of the efficacy and safety of cetirizine, oxatomide, ketotifen, and a placebo for the treatment of childhood perennial allergic rhinitis. Ann Allergy Asthma Immunol 2002;89(6):589-598.

(37) Chunharas A, Wisuthsarewong W, Wananukul S, Viravan S. Therapeutic efficacy and safety of loratadine syrup in childhood atopic dermatitis treated with mometasone furoate 0.1 per cent cream. J Med Assoc Thai 2002;85(4):482-487.

(38) La Rosa M, Leonardi S, Marchese G, Corrias A, Barberio G, Oggiano N, et al. Double-blind multicenter study on the efficacy and tolerability of cetirizine compared with oxatomide in chronic idiopathic urticaria in preschool children. Ann Allergy Asthma Immunol2001;87(1):48-53.

(39) Yang YH, Lin YT, Lu MY, Tsai MJ, Chiang BL. A double-blind, placebo-controlled, and randomized study of loratadine (Clarityne) syrup for the treatment of allergic rhinitis in children aged 3 to 12 years. Asian Pac J Allergy Immunol 2001;19(3):171-175.

(40) Ciprandi G, Tosca M, Passalacqua G, Canonica GW. Long-term cetirizine treatment reduces allergic symptoms and drug prescriptions in children with mite allergy. Ann Allergy Asthma Immunol2001;87(3):222226.

(41) Graft DF, Bernstein DI, Goldsobel A, Meltzer EO, Portnoy J, Long J. Safety of fexofenadine in children treated for seasonal allergic rhinitis. Ann Allergy Asthma Immunol 2001;87(1):22-26.

(42) Salmun LM, Herron JM, Banfield C, Padhi D, Lorber R, Affrime MB. The pharmacokinetics, electrocardiographic effects, and tolerability of loratadine syrup in children aged 2 to 5 years. Clin Ther2000;22(5):613621.

(43) Simons FE. Prospective, long-term safety evaluation of the H1-receptor antagonist cetirizine in very young children with atopic dermatitis. ETAC Study Group. Early Treatment of the Atopic Child.J Allergy Clin Immunol 1999;104(2 Pt 1):433-440.

(44) Allergic factors associated with the development of asthma and the influence of cetirizine in a doubleblind, randomised, placebo-controlled trial: first results of ETAC. Early Treatment of the Atopic Child. Pediatr Allergy Immunol 1998;9(3):116-124.

(45) Sienra-Monge JJ, Gazca-Aguilar A, Del Rio-Navarro B. Double-blind comparison of cetirizine and loratadine in children ages 2 to 6 years with perennial allergic rhinitis. Am J Ther 1999;6(3):149-155.

(46) Serra HA, Alves O, Rizzo LF, Devoto FM, Ascierto H. Loratadine-pseudoephedrine in children with allergic rhinitis, a controlled double-blind trial. Br J Clin Pharmacol1998;45(2):147-150.

(47) Delgado LF, Pferferman A, Sole D, Naspitz CK. Evaluation of the potential cardiotoxicity of the antihistamines terfenadine, astemizole, loratadine, and cetirizine in atopic children. Ann Allergy Asthma Immunol 1998;80(4):333-337.

(48) Pearlman DS, Lumry WR, Winder JA, Noonan MJ. Once-daily cetirizine effective in the treatment of seasonal allergic rhinitis in children aged 6 to 11 years: a randomized, double-blind, placebo-controlled study. Clin Pediatr (Phila) 1997;36(4):209-215.

(49) Winder JA, Noonan MJ, Lumry WR, Pearlman DS. Absence of QTc Prolongation with Cetirizine in Children Aged 6 to 11 Years.Pediatric Asthma, Allergy \& Immunology 1996;10(4):181-190.

(50) Ciprandi G, Tosca M, Ricca V, Passalacqua G, Riccio AM, Bagnasco M, et al. Cetirizine treatment of rhinitis in children with pollen allergy: evidence of its antiallergic activity. Clin Exp Allergy 1997;27(10):11601166 . 
(51) de Benedictis FM, Forenza N, Armenio L, Boner AL, Giorgi PL, del Giudice MM, et al. Efficacy and Safety of Cetirizine and Oxatomide in Young Children With Perennial Allergic Rhinitis: A 10-Day, Multicenter, Double-Blinded, Randomized, Parallel-Group Study. Pediatric Asthma, Allergy \&3 Immunology 1997;11(2):119-128.

(52) Tinkelman DG, Kemp J, Mitchell DQ, Galant SP. Treatment of Seasonal Allergic Rhinitis in Children with Cetirizine or Chlorpheniramine: A Multicenter Study. Pediatric Asthma, Allergy \& Immunology 1996;10(1):9-17.

(53) Fasce L, Ciprandi G, Pronzato C, Cozzani S, Tosca MA, Grimaldi I, et al. Cetirizine reduces ICAM-I on epithelial cells during nasal minimal persistent inflammation in asymptomatic children with mite-allergic asthma. Int Arch Allergy Immunol1996;109(3):272-276.

(54) La Rosa M, Ranno C, Musarra I, Guglielmo F, Corrias A, Bellanti JA. Double-blind study of cetirizine in atopic eczema in children. Ann Allergy 1994;73(2):117-122.

(55) Jobst S, van den Wijngaart W, Schubert A, van de Venne H. Assessment of the efficacy and safety of three dose levels of cetirizine given once daily in children with perennial allergic rhinitis. Allergy 1994;49(8):598-604.

(56) Allegra L, Paupe J, Wieseman HG, Baelde Y. Cetirizine for seasonal allergic rhinitis in children aged 2-6 years. A double-blind comparison with placebo. Pediatr Allergy Immunol 1993;4(3):157-161.

(57) Masi M, Candiani R, van de Venne H. A placebo-controlled trial of cetirizine in seasonal allergic rhinoconjunctivitis in children aged 6 to 12 years. Pediatr Allergy Immunol 1993;4(4 Suppl):47-52.

(58) Baelde,Y., Dupont,P. Cetirizine in Children with Chronic Allergic Rhinitis. A multicentre double-blind study of two doses of cetirizine and placebo. Drug Invest 1992; 4(6):466-472.

(59) Boner AL, Miglioranzi P, Richelli C, Marchesi E, Andreoli A. Efficacy and safety of loratadine suspension in the treatment of children with allergic rhinitis. Allergy 1989;44(6):437-441.

(60) Roberts G, Xatzipsalti M, Borrego LM, Custovic A, Halken S, Hellings PW, et al. Paediatric rhinitis: position paper of the European Academy of Allergy and Clinical Immunology. Allergy . 2013;68(9):11021116 .

(61) Brożek JL, Bousquet J, Agache I, Agarwal A, Bachert C, Bosnic-Anticevich S, et al. Allergic Rhinitis and its Impact on Asthma (ARIA) guidelines-2016 revision. J Allergy Clin Immunol . 2017;140(4):950-958.

(62) Church MK, Maurer M, Simons FE, Bindslev-Jensen C, van Cauwenberge P, Bousquet J, et al. Risk of first-generation H(1)-antihistamines: a GA(2)LEN position paper. Allergy . 2010;65(4):459-466.

Table 1. Trial characteristics

\begin{tabular}{|c|c|c|c|c|c|c|c|c|}
\hline $\begin{array}{l}\text { Study, } \\
\text { Year } \\
\text { (Reference) }\end{array}$ & Region & $\begin{array}{l}\text { Disease } \\
\text { type } \\
\text { (age } \\
\text { range) }\end{array}$ & $\begin{array}{l}\text { Treatment } \\
\text { groups }\end{array}$ & $\begin{array}{l}\text { Treatment } \\
\text { dose }\end{array}$ & $\begin{array}{l}\text { Treatment } \\
\text { dura- } \\
\text { tion, } \\
\text { weeks }\end{array}$ & $\begin{array}{l}\text { Patients, } \\
\mathrm{n}\end{array}$ & $\begin{array}{l}\text { Age, } \\
\text { years }\end{array}$ & $\begin{array}{l}\text { Female } \\
\%\end{array}$ \\
\hline $\begin{array}{l}\text { Wandalsen } \\
\text { et al., } \\
2017 \\
(13)\end{array}$ & $\begin{array}{l}\text { S. } \\
\text { America }\end{array}$ & $\begin{array}{l}\text { PAR } \\
(2-12 \\
\text { years })\end{array}$ & $\begin{array}{l}\text { DLRD } \\
\text { DCPN }\end{array}$ & $\begin{array}{l}1.25- \\
2.5 \mathrm{mg} \\
\text { po OD } \\
1-2 \mathrm{mg} \\
\text { po TD }\end{array}$ & 1 & 105105 & ND & 4546 \\
\hline $\begin{array}{l}\text { Nayak } \\
\text { et al., } \\
2017 \\
(14)\end{array}$ & USA & $\begin{array}{l}\text { SAR } \\
(6-11 \\
\text { years })\end{array}$ & $\begin{array}{l}\mathrm{CTZ} \\
\mathrm{LRD} \\
\text { placebo }\end{array}$ & $\begin{array}{l}10 \mathrm{mg} \text { po } \\
\text { OD } \\
10 \mathrm{mg} \text { po } \\
\text { OD }\end{array}$ & 2 & $\begin{array}{l}228220 \\
219\end{array}$ & $\begin{array}{l}8.68 .9 \\
8.9\end{array}$ & $\begin{array}{l}42.5 \\
42.2 \\
46.3\end{array}$ \\
\hline
\end{tabular}




\begin{tabular}{|c|c|c|c|c|c|c|c|c|}
\hline $\begin{array}{l}\text { Study, } \\
\text { Year } \\
\text { (Reference) } \\
\end{array}$ & Region & $\begin{array}{l}\text { Disease } \\
\text { type } \\
\text { (age } \\
\text { range) } \\
\end{array}$ & $\begin{array}{l}\text { Treatment } \\
\text { groups }\end{array}$ & $\begin{array}{l}\text { Treatment } \\
\text { dose }\end{array}$ & $\begin{array}{l}\text { Treatment } \\
\text { dura- } \\
\text { tion, } \\
\text { weeks }\end{array}$ & $\begin{array}{l}\text { Patients, } \\
\mathrm{n}\end{array}$ & $\begin{array}{l}\text { Age, } \\
\text { years }\end{array}$ & $\begin{array}{l}\text { Female } \\
\%\end{array}$ \\
\hline $\begin{array}{l}\text { Novak } \\
\text { et al., } \\
2016 \\
(15)\end{array}$ & $\begin{array}{l}\text { Europe, } \\
\text { S. } \\
\text { America }\end{array}$ & $\begin{array}{l}\text { Allergic } \\
\text { rhinocon- } \\
\text { juctivi- } \\
\text { tis or } \\
\text { chronic } \\
\text { urticar- } \\
\text { ial (2-12 } \\
\text { years) }\end{array}$ & $\begin{array}{l}\text { BLN } \\
\text { placebo }\end{array}$ & $\begin{array}{l}10 \mathrm{mg} \\
\text { po OD }\end{array}$ & 12 & 260249 & 7.57 .4 & $\begin{array}{l}27.3 \\
27.8\end{array}$ \\
\hline $\begin{array}{l}\text { Potter et } \\
\text { al., } 2016 \\
(16)\end{array}$ & Europe & $\begin{array}{l}\text { Chronic } \\
\text { sponta- } \\
\text { neous } \\
\text { urticaria } \\
\text { (2-11 years) }\end{array}$ & $\begin{array}{l}\text { RPD } \\
{[3] \mathrm{DLRD}} \\
\text { placebo }\end{array}$ & $\begin{array}{l}2.5 \mathrm{mg} \text { po } \\
\text { OD } \\
(10-25 \mathrm{~kg}) \\
5 \mathrm{mg} \text { po OD } \\
(>25 \mathrm{~kg}) \\
\text { standard } \\
\text { dose OD }\end{array}$ & 6 & $66[3] 7169$ & $\begin{array}{l}5.6[3] 6.1 \\
6.1\end{array}$ & $\begin{array}{l}56.1[3 \\
42\end{array}$ \\
\hline $\begin{array}{l}\text { Potter } \\
\text { et al. } \\
2013 \\
(17)\end{array}$ & $\begin{array}{l}\text { Europe } \\
\text { Africa } \\
\text { S. } \\
\text { America }\end{array}$ & $\begin{array}{l}\text { PAR } \\
(6-11 \\
\text { years })\end{array}$ & $\begin{array}{l}\mathrm{RPD} \\
\text { placebo }\end{array}$ & $\begin{array}{l}2,5 \mathrm{mg} \\
\text { po OD } \\
(<25 \mathrm{~kg}) \\
5 \mathrm{mg} \text { po } \\
\text { OD } \\
([?] 25 \mathrm{~kg})\end{array}$ & 6 & 180180 & 8.78 .8 & $\begin{array}{l}41.1 \\
38.3\end{array}$ \\
\hline $\begin{array}{l}\text { Wu et al., } \\
2012 \text { (18) }\end{array}$ & Asia & $\begin{array}{l}\text { PAR }(2-12 \\
\text { years) }\end{array}$ & $\begin{array}{l}\text { LRD } \\
\text { CPHD }\end{array}$ & $\begin{array}{l}5-10 \mathrm{mg} \text { po } \\
\text { OD } \\
(<30 \mathrm{~kg},>30 \mathrm{~kg} \\
2-4 \mathrm{mg} \text { po } \\
\text { TD } \\
(<30 \mathrm{~kg},>30 \mathrm{~kg}\end{array}$ & g) & 3030 & 5.86 .6 & 61.534 \\
\hline $\begin{array}{l}\text { Marcucci } \\
\text { et al., } \\
2011 \\
(19)\end{array}$ & Europe & $\begin{array}{l}\text { PAR } \\
(6-12 \\
\text { years })\end{array}$ & $\begin{array}{l}\text { LCTZ } \\
\text { placebo }\end{array}$ & $\mathrm{ND}$ & 12 & 3030 & $\begin{array}{l}9.73 \\
\text { (total) }\end{array}$ & $\begin{array}{l}60.0 \\
\text { (total) }\end{array}$ \\
\hline \multirow[t]{2}{*}{$\begin{array}{l}\text { Hampel } \\
\text { et al., } \\
2010 \\
(20)\end{array}$} & USA & $\begin{array}{l}\text { AR or } \\
\text { Chronic } \\
\text { urticaria } \\
\text { (6-11 } \\
\text { months) }\end{array}$ & $\begin{array}{l}\text { LCTZ } \\
\text { placebo }\end{array}$ & $\begin{array}{l}1.25 \mathrm{mg} \\
\text { po OD }\end{array}$ & 2 & 4524 & $\begin{array}{l}8.87 \\
\text { months } \\
9.03 \\
\text { months }\end{array}$ & $\begin{array}{l}37.8 \\
54.2\end{array}$ \\
\hline & & $\begin{array}{l}\text { AR or } \\
\text { Chronic } \\
\text { urticaria } \\
(1-5 \\
\text { years })\end{array}$ & $\begin{array}{l}\text { LCTZ } \\
\text { placebo }\end{array}$ & $\begin{array}{l}1.25 \mathrm{mg} \\
\text { po BD }\end{array}$ & 2 & 11459 & $\begin{array}{l}3.78 \\
3.75\end{array}$ & $\begin{array}{l}57.0 \\
61.0\end{array}$ \\
\hline $\begin{array}{l}\text { Lee et } \\
\text { al., } 2009 \\
(21)\end{array}$ & Europe & $\begin{array}{l}\text { PAR } \\
(6-12 \\
\text { years })\end{array}$ & $\begin{array}{l}\text { CTZ } \\
\text { LCTZ } \\
\text { placebo }\end{array}$ & $\begin{array}{l}10 \mathrm{mg} \text { po } \\
\text { OD } 5 \mathrm{mg} \\
\text { po OD }\end{array}$ & 12 & 272627 & $\begin{array}{l}8.19 \\
8.79 \\
8.12\end{array}$ & $\begin{array}{l}58.0 \\
62.5 \\
54.0\end{array}$ \\
\hline
\end{tabular}




\begin{tabular}{|c|c|c|c|c|c|c|c|c|}
\hline $\begin{array}{l}\text { Study, } \\
\text { Year } \\
\text { (Reference) }\end{array}$ & Region & $\begin{array}{l}\text { Disease } \\
\text { type } \\
\text { (age } \\
\text { range) }\end{array}$ & $\begin{array}{l}\text { Treatment } \\
\text { groups }\end{array}$ & $\begin{array}{l}\text { Treatment } \\
\text { dose }\end{array}$ & $\begin{array}{l}\text { Treatment } \\
\text { dura- } \\
\text { tion, } \\
\text { weeks }\end{array}$ & $\begin{array}{l}\text { Patients, } \\
\mathrm{n}\end{array}$ & $\begin{array}{l}\text { Age, } \\
\text { years }^{++}\end{array}$ & $\begin{array}{l}\text { Female, } \\
\%\end{array}$ \\
\hline \multicolumn{2}{|c|}{$\begin{array}{l}\text { NgamphaiboonAsia } \\
\text { et al., } \\
2009 \\
(22)\end{array}$} & $\begin{array}{l}\text { Recurrent } \\
\text { wheez- } \\
\text { ing ( }<6 \\
\text { years) }\end{array}$ & $\begin{array}{l}\text { LRD } \\
\text { KTF } \\
\text { placebo }\end{array}$ & $\begin{array}{l}0.25 \mathrm{ml} / \mathrm{kg} \\
\text { po OD } \\
0.25 \mathrm{ml} / \mathrm{kg} \\
\text { po OD }\end{array}$ & 16 & 272526 & $\begin{array}{l}3.22 .9 \\
2.9\end{array}$ & $\begin{array}{l}29.6 \\
52.0 \\
34.6\end{array}$ \\
\hline \multirow[t]{2}{*}{$\begin{array}{l}\text { Hampel } \\
\text { et al., } \\
2007 \\
(23)\end{array}$} & \multirow[t]{2}{*}{ USA } & $\begin{array}{l}\text { AR } \\
(6-12 \\
\text { months })\end{array}$ & $\begin{array}{l}\text { FXD } \\
\text { FXD } \\
\text { placebo }\end{array}$ & $\begin{array}{l}15 \mathrm{mg} \text { po } \\
\text { OD } \\
30 \mathrm{mg} \text { po } \\
\text { OD }\end{array}$ & 8 & 58569 & $\begin{array}{l}8.8 \\
\text { months } \\
10.4 \\
\text { months } \\
\text { ND }\end{array}$ & $\begin{array}{l}43.1 \\
40.0 \\
46.3\end{array}$ \\
\hline & & $\begin{array}{l}\text { AR }(1-2 \\
\text { years })\end{array}$ & $\begin{array}{l}\text { FXD } \\
\text { FXD } \\
\text { placebo }\end{array}$ & $\begin{array}{l}15 \mathrm{mg} \text { po } \\
\text { OD } \\
30 \mathrm{mg} \text { po } \\
\text { OD }\end{array}$ & 8 & $\begin{array}{l}27103 \\
131\end{array}$ & $\begin{array}{l}16.1 \\
\text { months } \\
17.9 \\
\text { months } \\
\text { ND }\end{array}$ & $\begin{array}{l}48.1 \\
39.8 \\
38.5\end{array}$ \\
\hline $\begin{array}{l}\text { Milgrom } \\
\text { et al., } \\
2007 \\
(24)\end{array}$ & USA & $\begin{array}{l}\mathrm{AR}(2-5 \\
\text { years })\end{array}$ & $\begin{array}{l}\text { FXD } \\
\text { placebo }\end{array}$ & $\begin{array}{l}30 \mathrm{mg} \text { po } \\
\text { OD }\end{array}$ & 2 & 222231 & 3.63 .6 & $\begin{array}{l}44.6 \\
44.9\end{array}$ \\
\hline $\begin{array}{l}\text { Simons } \\
\text { et al., } \\
2007 \\
(25)\end{array}$ & Canada & $\begin{array}{l}\text { AD } \\
(12-24 \\
\text { months })\end{array}$ & $\begin{array}{l}\text { LCTZ } \\
\text { placebo }\end{array}$ & $\begin{array}{l}0.125 \mathrm{mg} / \mathrm{kg} \\
\text { po OD }\end{array}$ & 72 & 255255 & $\begin{array}{l}19.3 \\
19.4\end{array}$ & $\begin{array}{l}39.2 \\
35.7\end{array}$ \\
\hline $\begin{array}{l}\text { Chen et al., } \\
2006(26)\end{array}$ & Taiwan & $\begin{array}{l}\text { PAR }(2-6 \\
\text { years })\end{array}$ & $\begin{array}{l}\text { CTZ MLK } \\
\text { placebo }\end{array}$ & $\begin{array}{l}5 \mathrm{mg} \text { ро OD } \\
4 \mathrm{mg} \text { ро OD }\end{array}$ & 12 & 202020 & 4.54 .54 .4 & $\begin{array}{l}40.045 \\
55.0\end{array}$ \\
\hline $\begin{array}{l}\text { Prenner } \\
\text { et al., } \\
2006 \\
(27)\end{array}$ & $\begin{array}{l}\text { USA, S. } \\
\text { Amer- } \\
\text { ica, S. } \\
\text { Africa }\end{array}$ & $\begin{array}{l}\text { ND } \\
(6-24 \\
\text { months })\end{array}$ & $\begin{array}{l}\text { DLRD } \\
\text { placebo }\end{array}$ & $\begin{array}{l}1.0 \mathrm{mg} \\
\text { po OD } \\
(<1 \mathrm{yr}) \\
1.25 \mathrm{mg} \\
\text { po OD } \\
(1-2 \mathrm{yrs})\end{array}$ & 2 & 131129 & $\begin{array}{l}12.6 \\
\text { months } \\
13.3 \\
\text { months }\end{array}$ & $\begin{array}{l}44.0 \\
53.0\end{array}$ \\
\hline $\begin{array}{l}\text { de Blic } \\
\text { et al., } \\
2005 \\
(28)\end{array}$ & Europe & $\begin{array}{l}\text { SAR } \\
(6-12 \\
\text { years })\end{array}$ & $\begin{array}{l}\text { LCTZ } \\
\text { placebo }\end{array}$ & $\begin{array}{l}5 \mathrm{mg} \text { po } \\
\text { OD }\end{array}$ & 6 & 8988 & 9.99 .9 & $\begin{array}{l}29.2 \\
38.6\end{array}$ \\
\hline $\begin{array}{l}\text { Potter } \\
\text { et al., } \\
2005 \\
(29)\end{array}$ & Africa & $\begin{array}{l}\text { PAR } \\
(6-12 \\
\text { years })\end{array}$ & $\begin{array}{l}\text { LCTZ } \\
\text { placebo }\end{array}$ & $\begin{array}{l}5 \mathrm{mg} \text { po } \\
\text { OD }\end{array}$ & 4 & 154152 & 9.99 .9 & $\begin{array}{l}35.7 \\
42.8\end{array}$ \\
\hline \multirow[t]{2}{*}{$\begin{array}{l}\text { Bloom } \\
\text { et al., } \\
2004 \\
(30)\end{array}$} & USA & $\begin{array}{l}\text { AR or } \\
\text { CIU } \\
(2-5 \\
\text { years })\end{array}$ & $\begin{array}{l}\text { DLRD } \\
\text { placebo }\end{array}$ & $\begin{array}{l}1.25 \mathrm{mg} \\
\text { po OD }\end{array}$ & 2 & 5556 & 3.53 .4 & $\begin{array}{l}44.0 \\
45.0\end{array}$ \\
\hline & & $\begin{array}{l}\text { AR or } \\
\text { CIU } \\
(6-11 \\
\text { years })\end{array}$ & $\begin{array}{l}\text { DLRD } \\
\text { placebo }\end{array}$ & $\begin{array}{l}2.5 \mathrm{mg} \\
\text { po OD }\end{array}$ & 2 & 6060 & 7.98 .5 & $\begin{array}{l}48.0 \\
65.0\end{array}$ \\
\hline
\end{tabular}




\begin{tabular}{|c|c|c|c|c|c|c|c|c|}
\hline $\begin{array}{l}\text { Study, } \\
\text { Year } \\
\text { (Reference) }\end{array}$ & Region & $\begin{array}{l}\text { Disease } \\
\text { type } \\
\text { (age } \\
\text { range) }\end{array}$ & $\begin{array}{l}\text { Treatment } \\
\text { groups }\end{array}$ & $\begin{array}{l}\text { Treatment } \\
\text { dose }\end{array}$ & $\begin{array}{l}\text { Treatment } \\
\text { dura- } \\
\text { tion, } \\
\text { weeks }\end{array}$ & $\begin{array}{l}\text { Patients, } \\
\mathrm{n}\end{array}$ & $\begin{array}{l}\text { Age, } \\
\text { years }\end{array}$ & $\begin{array}{l}\text { Female, } \\
\%\end{array}$ \\
\hline $\begin{array}{l}\text { Grimfeld } \\
\text { et al., } \\
2004 \\
(31)\end{array}$ & $\begin{array}{l}\text { Europe, } \\
\text { S. } \\
\text { Africa, } \\
\text { Asia, C. } \\
\text { and S. } \\
\text { America }\end{array}$ & $\begin{array}{l}\text { Rhinitis, } \\
\text { rhinopharyn- } \\
\text { gitis, } \\
\text { acute } \\
\text { otitis } \\
\text { media, } \\
\text { laryngi- } \\
\text { tis or } \\
\text { bronchi- } \\
\text { tis } \\
\text { (12-30 } \\
\text { months) }\end{array}$ & $\begin{array}{l}\text { LRD } \\
\text { placebo }\end{array}$ & $\begin{array}{l}2.5 \mathrm{mg} \\
\text { po OD } \\
([?] 2 \\
\text { yrs) } \\
5 \mathrm{mg} \text { po } \\
\text { OD (>2 } \\
\text { yrs) }\end{array}$ & 52 & 204208 & $\begin{array}{l}23.9 \\
\text { months } \\
24 \\
\text { months }\end{array}$ & $\begin{array}{l}35.8 \\
42.0\end{array}$ \\
\hline $\begin{array}{l}\text { Hsieh et } \\
\text { al., } 2004 \\
(32)\end{array}$ & Taiwan & $\begin{array}{l}\text { PAR } \\
(6-12 \\
\text { years })\end{array}$ & $\begin{array}{l}\text { CTZ } \\
\text { MLK } \\
\text { placebo }\end{array}$ & $\begin{array}{l}10 \mathrm{mg} \text { po } \\
\text { OD } 5 \mathrm{mg} \\
\text { po OD }\end{array}$ & 12 & 212122 & $\begin{array}{l}8.18 .2 \\
8.1\end{array}$ & $\begin{array}{l}40.0 \\
35.0 \\
45.0\end{array}$ \\
\hline $\begin{array}{l}\text { Wahn et } \\
\text { al., } 2003 \\
(33)\end{array}$ & $\begin{array}{l}\text { Europe, } \\
\text { Africa, } \\
\text { USA, } \\
\text { Australia }\end{array}$ & $\begin{array}{l}\text { SAR } \\
(6-11 \\
\text { years })\end{array}$ & $\begin{array}{l}\text { FXD } \\
\text { placebo }\end{array}$ & $\begin{array}{l}30 \mathrm{mg} \text { po } \\
\mathrm{BD}\end{array}$ & 2 & 464471 & 8.88 .8 & $\begin{array}{l}40.0 \\
33.5\end{array}$ \\
\hline $\begin{array}{l}\text { Segal et } \\
\text { al., } 2003 \\
(34)\end{array}$ & USA & $\begin{array}{l}\text { SAR } \\
(6-11 \\
\text { years })\end{array}$ & $\begin{array}{l}\text { CTZ } \\
\text { placebo }\end{array}$ & $\begin{array}{l}5 \mathrm{mg} \text { po } \\
\text { OD } \\
(<25 \mathrm{~kg}) \\
10 \mathrm{mg} \text { po } \\
\text { OD } \\
([?] 25 \mathrm{~kg})\end{array}$ & 2 & 8686 & 9.29 .1 & $\begin{array}{l}35.8 \\
37.3\end{array}$ \\
\hline $\begin{array}{l}\text { Simons } \\
\text { et al., } \\
2003 \\
(35)\end{array}$ & $\begin{array}{l}\text { N. } \\
\text { America }\end{array}$ & $\begin{array}{l}\text { AR, ur- } \\
\text { ticaria, } \\
\text { AD or } \\
\text { other } \\
\text { disorder } \\
\text { that } \\
\text { required } \\
\text { treat- } \\
\text { ment } \\
\text { with } \\
\mathrm{H}_{1-} \\
\text { antihistamines } \\
\text { (6-11 } \\
\text { months) }\end{array}$ & $\begin{array}{l}\text { CTZ } \\
\text { placebo }\end{array}$ & $\begin{array}{l}0.25 \mathrm{mg} / \mathrm{kg} \\
\text { po } \mathrm{BD}\end{array}$ & 1 & 4243 & $\begin{array}{l}8.5 \\
\text { months } \\
\text { (male), } \\
7.9 \\
\text { months } \\
\text { (female) } \\
8.0 \\
\text { months } \\
\text { (male), } \\
7.2 \\
\text { months } \\
\text { (female) }\end{array}$ & $\begin{array}{l}50.0 \\
53.5\end{array}$ \\
\hline $\begin{array}{l}\text { Lai et } \\
\text { al., } 2002 \\
(36)\end{array}$ & Asia & $\begin{array}{l}\text { PAR } \\
(6-12 \\
\text { years })\end{array}$ & $\begin{array}{l}\mathrm{CTZ} \\
\mathrm{OXD} \\
\mathrm{KTF} \\
\text { placebo }\end{array}$ & $\begin{array}{l}10 \mathrm{mg} \text { po } \\
\text { OD } 1 \mathrm{mg} \\
\text { po OD } \\
1 \mathrm{mg} \text { po } \\
\text { OD }\end{array}$ & 12 & $\begin{array}{l}202020 \\
20\end{array}$ & 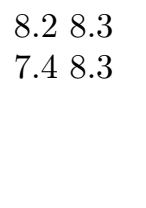 & $\begin{array}{l}58.7 \\
56.6 \\
56.2 \\
56.2\end{array}$ \\
\hline
\end{tabular}




\begin{tabular}{|c|c|c|c|c|c|c|c|c|}
\hline $\begin{array}{l}\text { Study, } \\
\text { Year } \\
\text { (Reference) }\end{array}$ & Region & $\begin{array}{l}\text { Disease } \\
\text { type } \\
\text { (age } \\
\text { range) }\end{array}$ & $\begin{array}{l}\text { Treatment } \\
\text { groups }\end{array}$ & $\begin{array}{l}\text { Treatment } \\
\text { dose }\end{array}$ & $\begin{array}{l}\text { Treatment } \\
\text { dura- } \\
\text { tion, } \\
\text { weeks }\end{array}$ & $\begin{array}{l}\text { Patients, } \\
\mathrm{n}\end{array}$ & $\begin{array}{l}\text { Age, } \\
\text { years }^{++}\end{array}$ & $\begin{array}{l}\text { Female, } \\
\%\end{array}$ \\
\hline $\begin{array}{l}\text { Chunharas } \\
\text { et al., } \\
2002 \\
(37)\end{array}$ & Asia & $\begin{array}{l}\text { AD } \\
(2-12 \\
\text { years })\end{array}$ & $\begin{array}{l}\text { LRD } \\
\text { placebo }\end{array}$ & $\begin{array}{l}5 \mathrm{ml} \text { po } \\
\text { OD } \\
([?] 30 \mathrm{~kg}) \\
10 \mathrm{ml} \text { po } \\
\text { OD } \\
(>30 \mathrm{~kg})\end{array}$ & 2 & 2424 & $\begin{array}{l}71.0 \\
\text { months } \\
76.3 \\
\text { months }\end{array}$ & $\begin{array}{l}54.0 \\
50.0\end{array}$ \\
\hline $\begin{array}{l}\text { La Rosa } \\
\text { et al., } \\
2001 \\
(38)\end{array}$ & Europe & $\begin{array}{l}\text { Chronic } \\
\text { urticaria } \\
(2-6 \\
\text { years })\end{array}$ & $\begin{array}{l}\text { OXD } \\
\text { CTZ }\end{array}$ & $\begin{array}{l}25 \mathrm{mg} \text { po } \\
\text { OD } 5 \mathrm{mg} \\
\text { po OD }\end{array}$ & 4 & 3131 & ND ND & ND ND \\
\hline $\begin{array}{l}\text { Yang et } \\
\text { al., } 2001 \\
(39)\end{array}$ & Asia & $\begin{array}{l}\text { AR } \\
(3-12 \\
\text { years })\end{array}$ & $\begin{array}{l}\text { LRD } \\
\text { placebo }\end{array}$ & $\begin{array}{l}5 \mathrm{mg} \text { po } \\
\text { OD } \\
(<30 \mathrm{~kg}) \\
10 \mathrm{mg} \text { po } \\
\text { OD } \\
([?] 30 \mathrm{~kg})\end{array}$ & 3 & 3030 & 6.06 .6 & $\begin{array}{l}36.4 \\
50.0\end{array}$ \\
\hline $\begin{array}{l}\text { Ciprandi } \\
\text { et al., } \\
2001 \\
(40)\end{array}$ & Europe & $\begin{array}{l}\text { Perennial } \\
\text { rhinocon- } \\
\text { junctivi- } \\
\text { tis, mild } \\
\text { inter- } \\
\text { mittent } \\
\text { asthma } \\
(3-10 \\
\text { years })\end{array}$ & $\begin{array}{l}\mathrm{CTZ} \\
\text { placebo }\end{array}$ & $\begin{array}{l}5 \mathrm{mg} \text { po } \\
\text { OD }\end{array}$ & 24 & 1010 & 6.26 .7 & $\begin{array}{l}30.0 \\
20.0\end{array}$ \\
\hline $\begin{array}{l}\text { Graft et al., } \\
2001(41)\end{array}$ & $\begin{array}{l}\text { USA, } \\
\text { Canada }\end{array}$ & $\begin{array}{l}\text { SAR }(6-11 \\
\text { years) }\end{array}$ & $\begin{array}{l}\text { FXD FXD } \\
\text { FXD } \\
\text { placebo }\end{array}$ & $\begin{array}{l}15 \mathrm{mg} \text { po } \\
\text { BD } 30 \mathrm{mg} \\
\text { po BD } \\
60 \mathrm{mg} \text { po } \\
\text { BD }\end{array}$ & 2 & $\begin{array}{l}223208212 \\
229\end{array}$ & $\begin{array}{ll}9.19 .19 .0 \\
9.2\end{array}$ & $\begin{array}{l}37.041 \\
47.0 \\
39\end{array}$ \\
\hline $\begin{array}{l}\text { Salmun } \\
\text { et al., } \\
2000 \\
(42)\end{array}$ & USA & $\begin{array}{l}\text { AR or } \\
\text { CIU } \\
(2-5 \\
\text { years })\end{array}$ & $\begin{array}{l}\text { LRD } \\
\text { placebo }\end{array}$ & $\begin{array}{l}5 \mathrm{mg} \text { po } \\
\text { OD }\end{array}$ & 2 & 6061 & 3.73 .5 & $\begin{array}{l}55.0 \\
55.7\end{array}$ \\
\hline $\begin{array}{l}\text { Simons } \\
\text { et al., } \\
1999 \\
(43) \\
\text { and } \\
\text { Wahn et } \\
\text { al., } 1998 \\
(44)^{+}\end{array}$ & $\begin{array}{l}\text { N. } \\
\text { America }\end{array}$ & $\begin{array}{l}\mathrm{AD}(1-2 \\
\text { years })\end{array}$ & $\begin{array}{l}\text { CTZ } \\
\text { placebo }\end{array}$ & $\begin{array}{l}0.25 \mathrm{mg} / \mathrm{kg} \\
\text { po } \mathrm{BD}\end{array}$ & 72 & 399396 & $\begin{array}{l}16.8 \\
\text { months } \\
17.2 \\
\text { months }\end{array}$ & $\begin{array}{l}38.1 \\
37.6\end{array}$ \\
\hline $\begin{array}{l}\text { Sienra- } \\
\text { Monge et } \\
\text { al., } 1999 \\
(45)\end{array}$ & C. America & $\begin{array}{l}\text { AR }(2-6 \\
\text { years })\end{array}$ & CTZ LRD & $\begin{array}{l}0.2 \mathrm{mg} / \mathrm{kg} \\
\text { po OD } \\
0.2 \mathrm{mg} / \mathrm{kg} \\
\text { po OD }\end{array}$ & 4 & 4040 & 4.34 .4 & 40.035 \\
\hline
\end{tabular}




\begin{tabular}{|c|c|c|c|c|c|c|c|c|}
\hline $\begin{array}{l}\text { Study, } \\
\text { Year } \\
\text { (Reference) }\end{array}$ & Region & $\begin{array}{l}\text { Disease } \\
\text { type } \\
\text { (age } \\
\text { range) }\end{array}$ & $\begin{array}{l}\text { Treatment } \\
\text { groups }\end{array}$ & $\begin{array}{l}\text { Treatment } \\
\text { dose }\end{array}$ & $\begin{array}{l}\text { Treatment } \\
\text { dura- } \\
\text { tion, } \\
\text { weeks }\end{array}$ & $\begin{array}{l}\text { Patients, } \\
\mathrm{n}\end{array}$ & $\begin{array}{l}\text { Age, } \\
\text { years }^{++}\end{array}$ & $\begin{array}{l}\text { Female, } \\
\%\end{array}$ \\
\hline $\begin{array}{l}\text { Serra et } \\
\text { al., } 1998 \\
(46)\end{array}$ & Argentina & $\begin{array}{l}\text { SAR } \\
(3-15 \\
\text { years })\end{array}$ & $\begin{array}{l}\text { LRD- } \\
\text { PSD } \\
\text { placebo }\end{array}$ & $\begin{array}{l}0.2 \mathrm{mg} / \mathrm{kg} \\
\text { po } \mathrm{BD}\end{array}$ & 2 & 2020 & ND ND & $\begin{array}{l}60.0 \\
60.0\end{array}$ \\
\hline $\begin{array}{l}\text { Delgado } \\
\text { et al., } \\
1998 \\
(47)\end{array}$ & Brazil & $\begin{array}{l}\text { PAR, } \\
\text { Sinusitis } \\
(5-12 \\
\text { years })\end{array}$ & $\begin{array}{l}\text { LRD } \\
\text { LRD } \\
\text { CTZ } \\
\text { CTZ }\end{array}$ & $\begin{array}{l}5 \mathrm{mg} \text { po } \\
\text { OD } \\
(<30 \mathrm{~kg}) \\
10 \mathrm{mg} \text { po } \\
\text { OD } \\
([?] 30 \mathrm{~kg}) \\
10 \mathrm{mg} \text { po } \\
\text { OD }\end{array}$ & 2 & $\begin{array}{l}101010 \\
10\end{array}$ & 7.29 .3 & $\begin{array}{l}50.0 \\
50.0 \\
50.0 \\
50.0\end{array}$ \\
\hline & USA & $\begin{array}{l}\text { AR } \\
(6-11 \\
\text { years })\end{array}$ & $\begin{array}{l}\mathrm{CTZ} \\
\mathrm{CTZ} \\
\text { placebo }\end{array}$ & $\begin{array}{l}5 \mathrm{mg} \text { po } \\
\text { OD } \\
10 \mathrm{mg} \text { po } \\
\text { OD }\end{array}$ & 4 & 697268 & $\begin{array}{l}9.18 .6 \\
8.8\end{array}$ & $\begin{array}{l}33.3 \\
27.1 \\
36.8\end{array}$ \\
\hline $\begin{array}{l}\text { Winder } \\
\text { et al., } \\
1996 \\
(49)^{+}\end{array}$ & & & & & & & & \\
\hline $\begin{array}{l}\text { Ciprandi } \\
\text { et al. } \\
1997 \\
(50)\end{array}$ & Europe & $\begin{array}{l}\text { Allergic } \\
\text { rhinocon- } \\
\text { junctivi- } \\
\text { tis ( } 6-15 \\
\text { years) }\end{array}$ & $\begin{array}{l}\text { CTZ } \\
\text { placebo }\end{array}$ & $\begin{array}{l}0.15 \mathrm{mg} / \mathrm{kg} \\
\text { po OD }\end{array}$ & 4 & 1010 & $\begin{array}{l}8.5 \\
\text { (total) }\end{array}$ & $\begin{array}{l}55.0 \\
\text { (total) }\end{array}$ \\
\hline $\begin{array}{l}\text { deBenedictis } \\
\text { et al., } \\
1997 \\
(51)\end{array}$ & Europe & $\begin{array}{l}\text { PAR } \\
(2-6 \\
\text { years })\end{array}$ & $\begin{array}{l}\text { CTZ } \\
\text { OXD }\end{array}$ & $\begin{array}{l}5 \mathrm{mg} \text { po } \\
\text { OD } \\
12 \mathrm{mg} \text { po } \\
\text { BD }\end{array}$ & $10 \mathrm{~d}$ & 5352 & 4.64 .8 & $\begin{array}{l}34.0 \\
40.0\end{array}$ \\
\hline $\begin{array}{l}\text { Tinkelman } \\
\text { et al. } \\
1996 \\
(52)\end{array}$ & USA & $\begin{array}{l}\text { SAR } \\
(6-11 \\
\text { years })\end{array}$ & $\begin{array}{l}\mathrm{CTZ} \\
\mathrm{CTZ} \\
\mathrm{CPN}\end{array}$ & $\begin{array}{l}5 \mathrm{mg} \text { po } \\
\text { OD } \\
(<25 \mathrm{~kg}) \\
10 \mathrm{mg} \text { po } \\
\text { OD } \\
([?] 25 \mathrm{~kg}) \\
2.5 \mathrm{mg} \\
\text { po BD } \\
(<25 \mathrm{~kg}) \\
5 \mathrm{mg} \text { po } \\
\text { BD } \\
([?] 25 \mathrm{~kg}) \\
2 \mathrm{mg} \text { po } \\
\text { TD }\end{array}$ & 2 & 636263 & $\begin{array}{l}8.69 .1 \\
8.7\end{array}$ & $\begin{array}{l}35.5 \\
29.5 \\
30.2\end{array}$ \\
\hline $\begin{array}{l}\text { Fasce et } \\
\text { al., } 1996 \\
(53)\end{array}$ & Europe & $\begin{array}{l}\text { Mite } \\
\text { allergic } \\
\text { asthma } \\
(5-14 \\
\text { years) }\end{array}$ & $\begin{array}{l}\text { CTZ } \\
\text { placebo }\end{array}$ & $\begin{array}{l}5 \mathrm{mg} \text { po } \\
\text { OD }\end{array}$ & 2 & 1010 & $\begin{array}{l}8.3 \\
\text { (total) }\end{array}$ & ND ND \\
\hline
\end{tabular}




\begin{tabular}{|c|c|c|c|c|c|c|c|c|}
\hline $\begin{array}{l}\text { Study, } \\
\text { Year } \\
\text { (Reference) }\end{array}$ & Region & $\begin{array}{l}\text { Disease } \\
\text { type } \\
\text { (age } \\
\text { range) }\end{array}$ & $\begin{array}{l}\text { Treatment } \\
\text { groups }\end{array}$ & $\begin{array}{l}\text { Treatment } \\
\text { dose }\end{array}$ & $\begin{array}{l}\text { Treatment } \\
\text { dura- } \\
\text { tion, } \\
\text { weeks }\end{array}$ & $\begin{array}{l}\text { Patients, } \\
\mathrm{n}\end{array}$ & $\begin{array}{l}\text { Age, } \\
\text { years }\end{array}$ & $\begin{array}{l}\text { Female, } \\
\%\end{array}$ \\
\hline $\begin{array}{l}\text { LaRosa } \\
\text { et al., } \\
1994 \\
(54)\end{array}$ & Europe & $\begin{array}{l}\text { AD } \\
(6-12 \\
\text { years })\end{array}$ & $\begin{array}{l}\text { CTZ } \\
\text { placebo }\end{array}$ & $\begin{array}{l}5 \mathrm{mg} \text { po } \\
\text { OD } \\
([?] 30 \mathrm{~kg}) \\
10 \mathrm{mg} \mathrm{po} \\
\text { OD } \\
(>30 \mathrm{~kg})\end{array}$ & 8 & 1211 & $\begin{array}{l}7.0 \\
\text { (total) }\end{array}$ & $\begin{array}{l}41.6 \\
63.6\end{array}$ \\
\hline $\begin{array}{l}\text { Jobst et } \\
\text { al., } 1994 \\
(55)\end{array}$ & Europe & $\begin{array}{l}\text { PAR } \\
(6-12 \\
\text { years })\end{array}$ & $\begin{array}{l}\mathrm{CTZ} \\
\mathrm{CTZ} \\
\mathrm{CTZ} \\
\text { placebo }\end{array}$ & $\begin{array}{l}2.5 \mathrm{mg} \\
\text { po OD } \\
5 \mathrm{mg} \text { po } \\
\text { OD } \\
10 \mathrm{mg} \text { po } \\
\text { OD }\end{array}$ & 2 & $\begin{array}{l}848576 \\
83\end{array}$ & $\begin{array}{ll}8.6 & 9.2 \\
9.3 & 8.9\end{array}$ & $\begin{array}{l}45.2 \\
29.4 \\
42.1 \\
45.8\end{array}$ \\
\hline $\begin{array}{l}\text { Allegra } \\
\text { et al., } \\
1993 \\
(56)\end{array}$ & Europe & $\begin{array}{l}\text { SAR } \\
(2-6 \\
\text { years })\end{array}$ & $\begin{array}{l}\mathrm{CTZ} \\
\text { placebo }\end{array}$ & $\begin{array}{l}5 \mathrm{mg} \text { po } \\
\text { OD }\end{array}$ & 2 & 5453 & 4.64 .3 & $\begin{array}{l}33.3 \\
28.3\end{array}$ \\
\hline $\begin{array}{l}\text { Masi et } \\
\text { al., } 1993 \\
(57)\end{array}$ & Europe & $\begin{array}{l}\text { Seasonal } \\
\text { allergic } \\
\text { rhinocon- } \\
\text { junctivi- } \\
\text { tis (6-12 } \\
\text { years) }\end{array}$ & $\begin{array}{l}\text { CTZ } \\
\text { placebo }\end{array}$ & $\begin{array}{l}5 \mathrm{mg} \text { po } \\
\mathrm{BD}\end{array}$ & 2 & 6361 & $\begin{array}{l}10.1 \\
10.2\end{array}$ & $\begin{array}{l}39.7 \\
37.7\end{array}$ \\
\hline $\begin{array}{l}\text { Baelde } \\
\text { et al., } \\
1992 \\
(58)\end{array}$ & Europe & $\begin{array}{l}\text { PAR } \\
(2-14 \\
\text { years })\end{array}$ & $\begin{array}{l}\text { CTZ } \\
\text { CTZ } \\
\text { placebo }\end{array}$ & $\begin{array}{l}5 \mathrm{mg} \text { po } \\
\mathrm{BD} \\
2.5 \mathrm{mg} \\
\text { po BD }\end{array}$ & 2 & 464646 & $\begin{array}{l}8.88 .5 \\
8.6\end{array}$ & $\begin{array}{l}30.4 \\
30.4 \\
39.1\end{array}$ \\
\hline $\begin{array}{l}\text { Boner et } \\
\text { al., } 1989 \\
(59)\end{array}$ & Europe & $\begin{array}{l}\text { SAR } \\
(4-12 \\
\text { years })\end{array}$ & $\begin{array}{l}\mathrm{LRD} \\
\mathrm{DCPN}\end{array}$ & $\begin{array}{l}5 \mathrm{mg} \text { po } \\
\text { OD } \\
\text { ([?]20kg } \\
\text { or [?]6 } \\
\text { yrs), } \\
2.5 \mathrm{mg} \\
\text { po OD } \\
(<20 \mathrm{~kg} \\
\text { or } \\
<6 \mathrm{yrs}) \\
1 \mathrm{mg} \text { po } \\
\mathrm{TD} \\
([?] 20 \mathrm{~kg} \\
\text { or [?]6 } \\
\text { yrs), } \\
0.5 \mathrm{mg} \\
\text { po TD } \\
(<20 \mathrm{~kg} \\
\text { or } \\
<6 \mathrm{yrs})\end{array}$ & 2 & 2119 & 7.67 .8 & $\begin{array}{l}33.3 \\
36.8\end{array}$ \\
\hline
\end{tabular}


Abbreviations: $\mathrm{AD}=$ Atopic dermatitis; $\mathrm{AR}=$ Allergic rhinitis; $\mathrm{BD}=\mathrm{Bis}$ in die (twice daily); $\mathrm{BLN}=$ Bilastine; $\mathrm{CIU}=$ Chronic idiopathic urticaria; $\mathrm{CPHD}=$ Cyproheptadine; $\mathrm{CPN}=$ Chlorpheniramine; $\mathrm{CTZ}=$ Cetirizine; DCPN = Dexchlorpheniramine; DLRD = Desloratadine; FXD = Fexofenadine; KTF = Ketotifen; $\mathrm{LCTZ}=$ Levocetirizine; $\mathrm{LRD}=$ Loratadine $\mathrm{MLK}=$ Montelukast $\mathrm{ND}=$ No data $\mathrm{OD}=$ Omne in die (once daily); $\mathrm{OXD}=$ Oxotamide; $\mathrm{PAR}=$ Perennial allergic rhinitis; $\mathrm{PO}=$ Per os $; \mathrm{PSD}=$ Pseudoephedrine; $\mathrm{QD}$ $=$ Quater in die (four times daily); RPD $=$ Rupatadine; $\mathrm{SAR}=$ Seasonal allergic rhinitis; $\mathrm{TD}=$ Ter in die (three times daily). $\mathrm{CPHD}=$ cyproeptadine + Two publications of the same RCT. ++ Data are mean years unless otherwise indicated.

Table 2. Risk of bias in included trials

\begin{tabular}{|c|c|c|c|}
\hline Study, Year (Reference) & Sequence generation & Allocation concealment & Blinding \\
\hline Wandalsen et al., 2017 (13) & Unclear & Unclear & Low \\
\hline Nayak et al., 2017 (14) & Unclear & Unclear & Unclear \\
\hline Novak et al., 2016 (15) & Low & Unclear & Low \\
\hline Potter et al., 2016 (16) & Unclear & Unclear & Low \\
\hline Potter et al., 2013 (17) & Unclear & Unclear & Low \\
\hline Wu et al., $2012(18)$ & Unclear & High & High \\
\hline Marucci et al., 2011 (19) & Unclear & Low & Unclear \\
\hline Hampel et al., 2010 (20) & Unclear & Unclear & Low \\
\hline Lee et al., $2009(21)$ & Unclear & Unclear & Unclear \\
\hline Ngamphaiboon et al., 2009 (22) & Unclear & Unclear & Unclear \\
\hline Hampel et al., 2007 (23) & Unclear & Unclear & Unclear \\
\hline Milgrom et al., 2007 (24) & Unclear & Unclear & Low \\
\hline Simons et al., 2007 (25) & Unclear & Unclear & Low \\
\hline Chen et al., 2006 (26) & Unclear & Unclear & Unclear \\
\hline Prenner et al., 2006 (27) & Low & Unclear & Low \\
\hline de Blic et al., 2005 (28) & Unclear & Unclear & Low \\
\hline Potter et al., 2005 (29) & High & Low & Low \\
\hline Bloom et al., 2004 (30) & Unclear & Unclear & Low \\
\hline Grimfeld et al., 2004 (31) & Unclear & Unclear & Unclear \\
\hline Hsieh et al., 2004 (32) & Low & Unclear & Unclear \\
\hline Wahn et al., 2003 (33) & Unclear & Unclear & Low \\
\hline Segal et al., 2003 (34) & Unclear & Unclear & Unclear \\
\hline Simons et al., 2003 (35) & Unclear & Unclear & Low \\
\hline Lai et al., $2002(36)$ & Low & Unclear & Unclear \\
\hline Chunharas et al., 2002 (37) & Unclear & Unclear & Unclear \\
\hline La Rosa et al., 2001 (38) & Low & Low & Low \\
\hline Yang et al., 2001 (39) & Unclear & Unclear & Unclear \\
\hline Ciprandi et al., 2001 (40) & Low & Unclear & Low \\
\hline Graft et al., 2001 (41) & Unclear & Unclear & Unclear \\
\hline Salmun et al., $2000(42)$ & Unclear & Unclear & Unclear \\
\hline Simons et al., 1999 (43) and Wahn et al., 1998 (44) & Low & Low & Low \\
\hline Sienra-Monge et al., 1999 (45) & Unclear & Unclear & Unclear \\
\hline Serra et al., 1998 (46) & Unclear & Unclear & Unclear \\
\hline Delgado et al., 1998 (47) & Unclear & Unclear & Unclear \\
\hline Pearlman et al., 1997 (48) and Winder et al., 1996 (49) & Unclear & Unclear & Low \\
\hline Ciprandi et al., 1997 (50) & Unclear & Unclear & Unclear \\
\hline deBenedictis et al., 1997 (51) & Unclear & Unclear & Low \\
\hline Tinkelman et al., 1996 (52) & Unclear & Low & High \\
\hline Fasce et al., $1996(53)$ & Unclear & Unclear & Unclear \\
\hline La Rosa et al., 1994 (54) & Unclear & Unclear & Low \\
\hline
\end{tabular}




\begin{tabular}{llll}
\hline Study, Year (Reference) & Sequence generation & Allocation concealment & Blinding \\
\hline Jobst et al., $1994(55)$ & Low & Unclear & Low \\
Allegra et al., $1993(56)$ & Low & Unclear & Low \\
Masi et al., $1993(57)$ & Unclear & Unclear & Low \\
Baelde et al., $1992(58)$ & Low & Unclear & Low \\
Boner et al. $1989(59)$ & Unclear & Unclear & High \\
\hline
\end{tabular}

Table 3. Adverse events and treatment discontinuations

\begin{tabular}{|c|c|c|c|c|c|c|c|}
\hline $\begin{array}{l}\text { Study, } \\
\text { Year } \\
\text { (Refer- } \\
\text { ence) }\end{array}$ & $\begin{array}{l}\text { Treatment } \\
\text { groups } \\
\text { (n) }\end{array}$ & $\begin{array}{l}\text { Patients, } \\
\text { n }\end{array}$ & $\begin{array}{l}\text { Patients } \\
\text { with } \\
\text { serious } \\
\text { AEs, \% }\end{array}$ & $\begin{array}{l}\text { Patients } \\
\text { with [?]1 } \\
\text { AE, \% }\end{array}$ & $\begin{array}{l}\text { Patients } \\
\text { with AEs } \\
\text { related } \\
\text { to study } \\
\text { drug, } \\
\%+\end{array}$ & $\begin{array}{l}\text { Treatment } \\
\text { discon- } \\
\text { tinua- } \\
\text { tions due } \\
\text { to AEs, } \\
\%\end{array}$ & $\begin{array}{l}\text { Comments } \\
\text { in } \\
\text { original } \\
\text { trials }\end{array}$ \\
\hline $\begin{array}{l}\text { Wandalsen } \\
\text { et al., } \\
2017 \text { (13) }\end{array}$ & $\begin{array}{l}\text { DLRD } \\
\text { DCPN }\end{array}$ & 105105 & ND ND & ND ND & ND ND & 0.91 .9 & $\begin{array}{l}\text { Six } \\
\text { patients in } \\
\text { the DLRD } \\
\text { group and } \\
11 \text { in the } \\
\text { DCPN } \\
\text { group } \\
\text { presented } \\
\text { ECG } \\
\text { changes at } \\
\text { the end of } \\
\text { the study }\end{array}$ \\
\hline $\begin{array}{l}\text { Nayak et } \\
\text { al., } 2017 \\
(14)\end{array}$ & $\begin{array}{l}\text { CTZ LRD } \\
\text { placebo }\end{array}$ & $\begin{array}{l}228220 \\
219\end{array}$ & $\begin{array}{llll}0 & 0 & 0\end{array}$ & $\begin{array}{l}19.721 .8 \\
22.7\end{array}$ & 4.84 .52 .6 & 2.65 .63 .9 & \\
\hline $\begin{array}{l}\text { Novak et al, } \\
2016(15)\end{array}$ & $\begin{array}{l}\text { BLN } \\
\text { placebo }\end{array}$ & 260249 & 0.73 .6 & 68.567 .5 & 5.88 .0 & 1.10 .8 & $\begin{array}{l}\text { No clinically } \\
\text { and/or } \\
\text { statistically } \\
\text { relevant } \\
\text { differences } \\
\text { between } \\
\text { bilastine and } \\
\text { placebo for } \\
\text { vital signs, } \\
\text { clinical } \\
\text { laboratory } \\
\text { values, ECG } \\
\text { parameters } \\
\text { or physical } \\
\text { examination }\end{array}$ \\
\hline $\begin{array}{l}\text { Potter et } \\
\text { al., } 2016 \\
(16)\end{array}$ & $\begin{array}{l}\text { RPD } \\
\text { DLRD } \\
\text { placebo }\end{array}$ & 667169 & $\begin{array}{l}\text { ND ND } \\
\text { ND }\end{array}$ & $\begin{array}{l}61.065 .0 \\
54.0\end{array}$ & 1.55 .67 .2 & $\begin{array}{l}\text { ND ND } \\
\text { ND }\end{array}$ & \\
\hline $\begin{array}{l}\text { Potter et } \\
\text { al., } 2013 \\
(17)\end{array}$ & $\begin{array}{l}\text { RPD } \\
\text { placebo }\end{array}$ & 180180 & ND ND & 37.230 .0 & 6.15 .5 & ND ND & \\
\hline
\end{tabular}




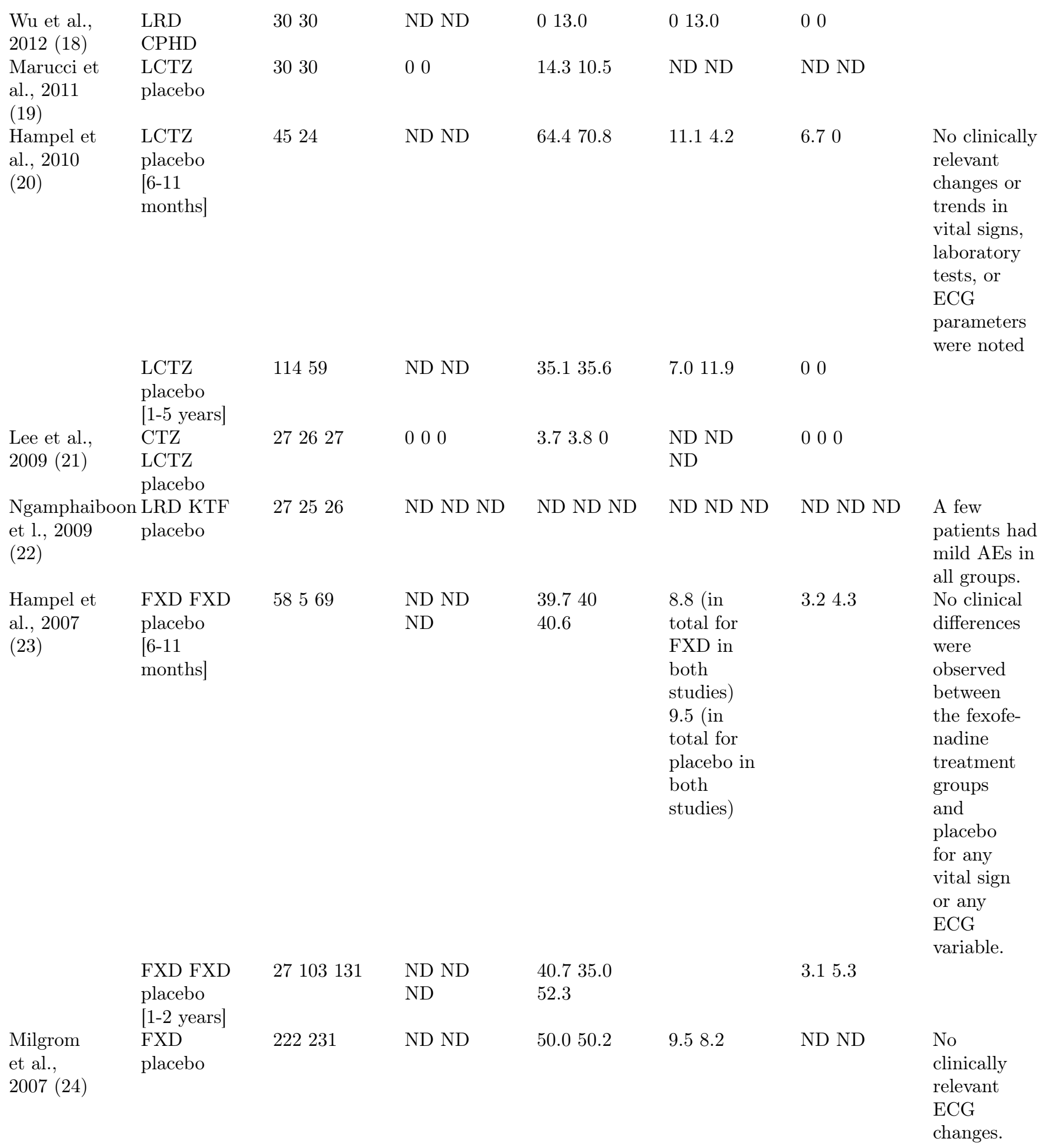


Simons et al., 2007

(25)

Chen et

al., 2006

(26)

Prenner et

al., 2006

(27)

de Blic et

al., 2005

(28)

Potter et

al., 2005

(29)

\section{LCTZ}

placebo
CTZ MLK

placebo

DLRD

placebo
255255

(1)

5.16 .3

202020

131129

131129

LCTZ

placebo

LCTZ

placebo
8988

154152
000

00.8

67.258 .9

2621.8

0.81 .6

00

00
5.65 .6

01.0

55.252 .6
000

10.000

1.8

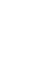

2.01 .2 
Bloom et

al., 2004

(30)

\begin{abstract}
DLRD
\end{abstract}
placebo
5556

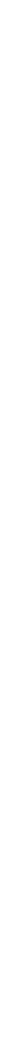

Grimfeld

et al., 2004 (31)

Hsieh et

al., 2004

(32)
DLRD

placebo

LRD

placebo

6060

204208

CTZ MLK

placebo
ND ND

12.710 .7

3.60

00

ND ND

ND ND

1.710 .0

00

00

80.985 .0

ND ND

00.5

5.05 .0

10.0
No

clinically relevant changes

were noted in median clinical laboratory test values or mean vital signs. Increase in heart rate was observed in the study of subjects aged 2 years-5 years, but the incidence was similar in the desloratadine and placebo groups.

There was no difference between groups regarding ECG or laboratory parameters. 
Wahn et

al., 2003

(33)

Segal et

al., 2003

(34)

Simons et al., 2003

(35)

Lai et al

2002 (36)

Chunharas

et al.,

2002 (37)
FXD

placebo

464471

$0.2 \mathrm{ND}$

placebo

CTZ

placebo

CTZ

placebo

4243

ND ND

73.888 .4

45.262 .8

7.16 .9

32.120 .9

ND ND

00

0.60

There

were no significant differences in changes in clinical laboratory and vital signs results between the fexofenadineand placebotreated groups.

All cause and treatment related AEs were generally more frequent in the 6to 8month age group than in the 9 to 11-month age group. 


\begin{tabular}{|c|c|c|c|c|c|c|c|}
\hline $\begin{array}{l}\text { La Rosa } \\
\text { et al., } \\
2001(38)\end{array}$ & CTZ OXT & 3131 & ND ND & 03.2 & 03.2 & 03.2 & $\begin{array}{l}\text { The hema- } \\
\text { tologic, } \\
\text { hemato- } \\
\text { chemical, } \\
\text { and } \\
\text { urinary } \\
\text { tests were } \\
\text { within the } \\
\text { normal } \\
\text { limits in } \\
\text { all } \\
\text { patients } \\
\text { before and } \\
\text { at the end } \\
\text { of the } \\
\text { treatment. }\end{array}$ \\
\hline $\begin{array}{l}\text { Yang et } \\
\text { al., } 2001 \\
(39)\end{array}$ & $\begin{array}{l}\text { LRD } \\
\text { placebo }\end{array}$ & 3030 & ND ND & 00 & 00 & ND ND & \\
\hline $\begin{array}{l}\text { Ciprandi } \\
\text { et al., } \\
2001(40)\end{array}$ & $\begin{array}{l}\text { CTZ } \\
\text { placebo }\end{array}$ & 1010 & ND ND & 00 & 00 & 00 & \\
\hline Graft et & FXD FXD & 223208 & ND ND & 35.336 .8 & ND ND & 0.401 .40 & Lab - \\
\hline $\begin{array}{l}\text { al., } 2001 \\
(41)\end{array}$ & $\begin{array}{l}\text { FXD } \\
\text { placebo }\end{array}$ & 212229 & ND ND & 34.736 .2 & ND ND & 0.502 .20 & $\begin{array}{l}\text { ECG } \\
\text { changes } \\
\text { unremark- } \\
\text { able, vital } \\
\text { signs } \\
\text { consistent } \\
\text { among } \\
\text { groups. }\end{array}$ \\
\hline
\end{tabular}


Salmun et al., 2000

(42)

Simons et al., 1999

(43) and Wahn et al., 1998

(44)

\section{Sienra-}

Monge et

al.,1999

(45)

Serra et

al., 1998

(46)
LRD

placebo

6061

placebo

CTZ

placebo

399396

placebo

CTZ LRD

4040

LRD-PSD

2020

placebo

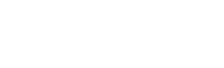

ND ND

32.041 .0

ND ND

00

98.598 .7

9.313 .6

0.51 .8

2.83 .8 


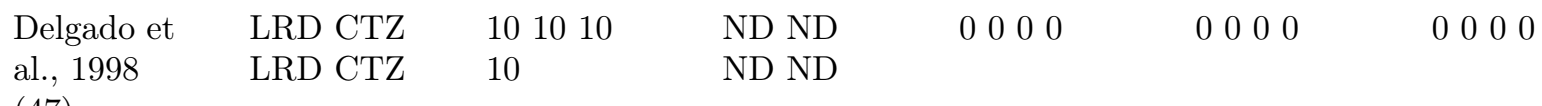

Pearlman et al., 1997 (48) and Winder et al., 1996 (49)

Ciprandi

et al., 1997 (50) Benedictis et al., 1997 (51)

Tinkelman et al., 1996 (52)
CTZ

placebo

CTZ OXD

5352

CTZ CTZ $\mathrm{CPN}$

1010

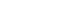

ND ND

8.07 .7

ND ND
ND ND
ND

33.638 .1
00

ND ND ND

00

000
No clinically significant changes in laboratory tests were found except for one oxatomide patient. 
Fasce et

al., 1996

(53)

La Rosa

et al.,

1994 (54)

Jobst et

al., 1994

(55)

Allegra et

al.,1993

(56)

Masi et

al., 1993

(57)
CTZ

placebo

1010

CTZ

placebo

CTZ CTZ

CTZ

placebo

CTZ

placebo

1211

848576

83

5453

CTZ

placebo

6361
ND ND

ND ND

ND ND

00

ND ND

ND ND

$0 \mathrm{ND}$

0 ND

ND ND

25.014 .0

ND ND

22.418 .4

ND ND

4.82 .91 .3

ND ND

24.120 .7

5.60

1.83 .8

ND ND

.

5.6

ND ND

22.222 .9

ND ND

3.21 .6
No important adverse reactions reported.

Minor changes in clinical laboratory tests were carefully reviewed and considered not to be clinically relevant. Minor changes in clinical laboratory tests were considered not to be clinically relevant. 
Baelde et

al., 1992

(58)

Boner et

al., 1989

(59)
CTZ CTZ 464646

Placebo
ND ND ND

6.517 .313 .0

ND ND ND

000

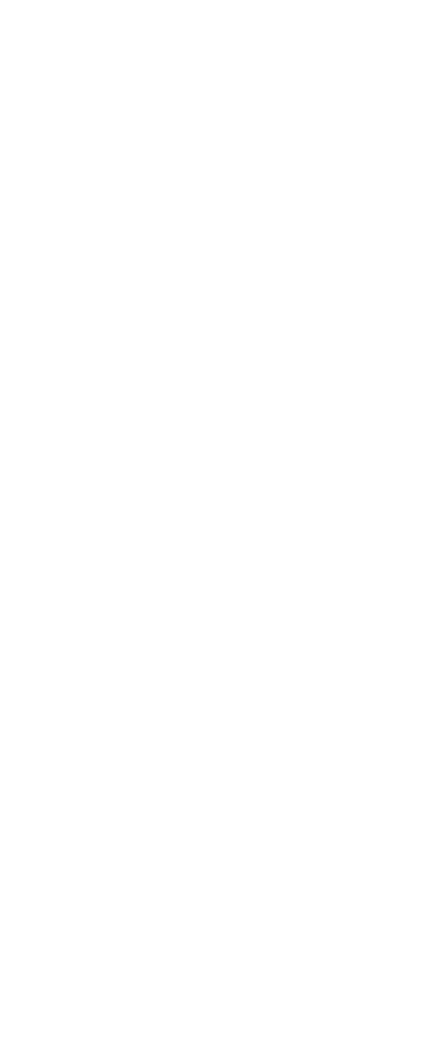

LRD

DCPN
2119

ND

19.031 .5

ND

4.70

D.

Leucocytosis

that occurred in some

patients in the three groups was not considered clinically relevant, a slight and clinically unimportant increase in AST levels with no effect on the liver's function occurred in some patients in the three groups, no clinically significant changes in blood urea or creatinine levels.

Hematological counts and biochemical analysis showed that loratadine and dexchlorpheniramine at the dosages used were devoid of toxic effects.

Abbreviations: $\mathrm{AE}=$ Adverse event $\mathrm{BLN}=$ Bilastine; $\mathrm{CPHD}=$ Cyproheptadine; $\mathrm{CPN}=$ Chlorpheniramine; 
$\mathrm{CTZ}=$ Cetirizine DCPN $=$ Dexchlorpheniramine DLRD $=$ Desloratadine $; \mathrm{FXD}=$ Fexofenadine KTF $=$ Ketotifen; LCTZ $=$ Levocetirizine; LRD $=$ Loratadine; MLK $=$ Montelukast; $\mathrm{ND}=$ No data; $\mathrm{OXD}=$ Oxotamide; PSD = Pseudoephedrine; RPD = Rupatadine; + Relation to study drug was assessed by the authors of each trial.

Table 4. Adverse events reported in included RCTs

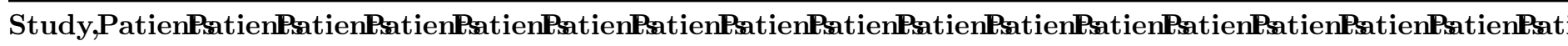
Year with with with with with with with with with with with with with with with with with (Ref- Ad- Ad- Ad- Ad- Ad- Ad- Ad- Ad- Ad- Ad- Ad- Ad- Ad- Ad- Ad- Ad- Ader- verse verse verse verse verse verse verse verse verse verse verse verse verse verse verse verse vers ence) Event\$vent\$vent\$vent\$vent\$vent\$vent\$vent\$vent\$vent\$vent\$vent\$ventEvent\$vent\$vent\$ve

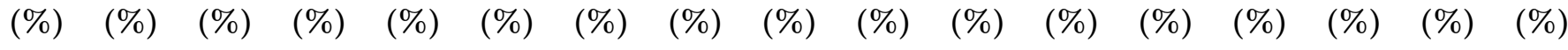
per per per per per per per per per per per per per per per per per treat- treat- treat- treat- treat- treat- treat- treat- treat- treat- treat- treat- treat- treat- treat- treat- trea ment ment ment ment ment ment ment ment ment ment ment ment ment ment ment ment mer group group group group group group group group group group group group group group group group grol WandaIßnD DLRD DLRD DLRD DLRD DLRD DCPN DCPN DCPN DCPN DCPN DCPN

et

al.,

2017

(13) 


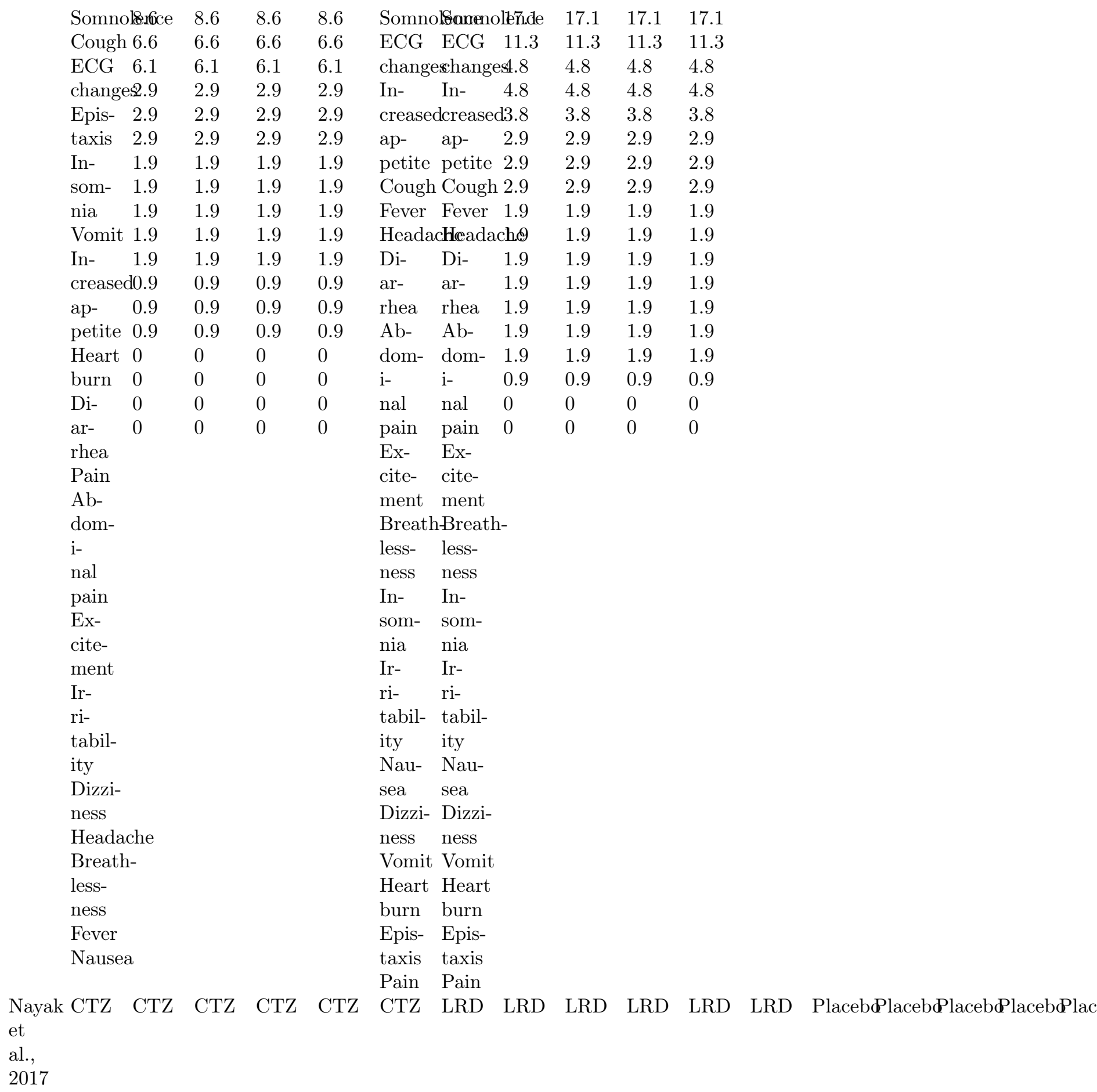




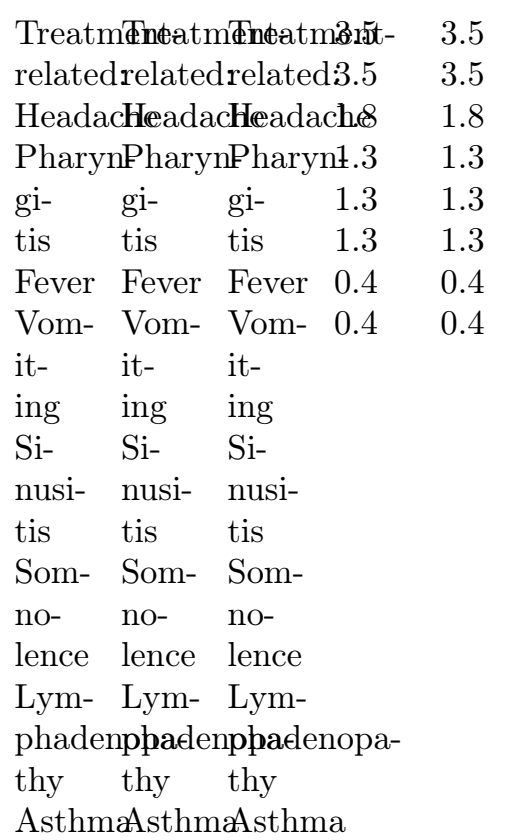

Novak BLN BLN BLN

et

al.,

2016

(15)

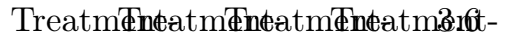
related related related related 2.7

Headacheadacheadacheadache

PharynPharynPharynPharynt. 8

gi- gi- gi- gi- 1.8

tis tis tis tis 1.8

Vom- Vom- Vom- Vom- 1.4

it- it- it- it- 0.9

ing ing ing ing 0.5

Si- Si- Si- Si-

nusi- nusi- nusi- nusi-

tis tis tis tis

Lym- Lym- Lym- Lym-

phadenppadenppadenppadenopa-

thy thy thy thy

Nau- Nau- Nau- Nau-

sea sea sea sea

Epis- Epis- Epis- Epis-

taxis taxis taxis taxis

Fever Fever Fever Fever

AsthmAsthmAsthmAsthma

PlacebPlaceb\&laceb\&lacebo
Treatmentatmineatmintrelatedrelated related 3.5

Headacheadacheadach $\bar{\theta}$

PharynPharynPharynt.3

gi- gi- gi- 1.3

tis tis tis 0.9

Si- Si- Si- 0.9

nusi- nusi- nusi- 0.9

tis tis tis 0.9

Asthmasthmasthma

Bron- Bron- Bron-

chi- chi- chi-

tis tis tis

Fever Fever Fever

Vom- Vom- Vom-

it- it- it-

ing ing ing

Nau- Nau- Nau-

sea sea sea

Epistaxtippistaxtispistaxis
3.1

3.5

1.7

1.3

1.3

0.9

0.9

0.9

0.9 


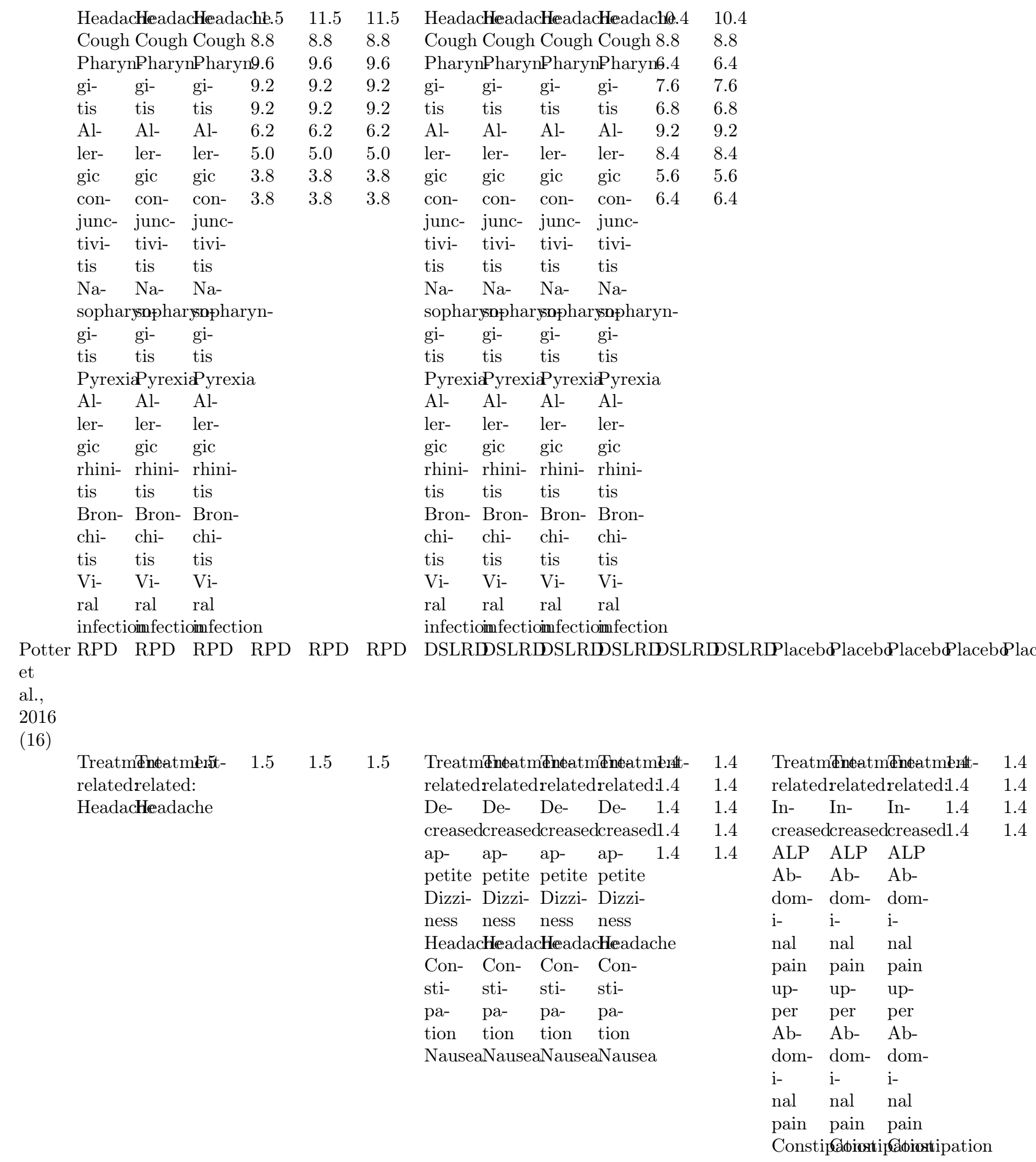


Potter RPD RPD RPD RPD RPD RPD Placebهlaceb\&lacebهlacebdlacebهlacebo et

al.,

2013

(17)

$\begin{array}{lllllll}\text { Headacheadacheadache. } 8 & 12.8 & 12.8 & \text { Headacheadac牙 } 6 & 5.6 & 5.6 & 5.6\end{array}$

Cough,Cough,Cough, $<4.0<4.0<4.0 \quad$ Cough, Cough, $<4.0<4.0<4.0<4.0$

Ab- Ab- Ab- $1.1 \quad 1.1 \quad 1.1 \quad$ Ab- Ab-

dom- dom- dom- dom- dom-

i- i- i- i- i-

nal nal nal nal nal

pain, pain, pain, pain, pain,

Epis- Epis- Epis- Epis- Epis-

taxis, taxis, taxis, taxis, taxis,

EczemaEczemałczema, Eczematczema,

In- In- In- Influenæafluenza

fluenzafluenzafluenza

Somnoldomenoldamenolence

Wu LRD LRD LRD LRD LRD LRD CPHD CPHD CPHD CPHD CPHD CPHD

et

al.,

2012

(18)

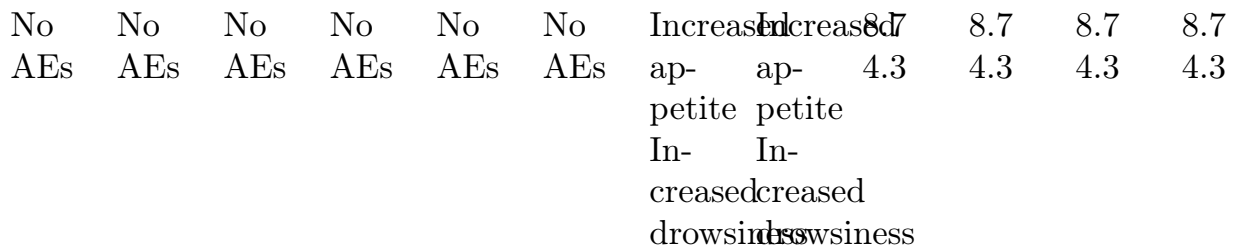

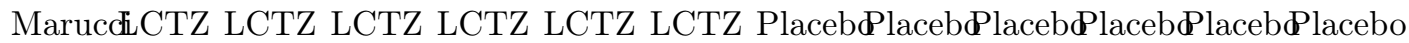
et

al.,

2011

(19)

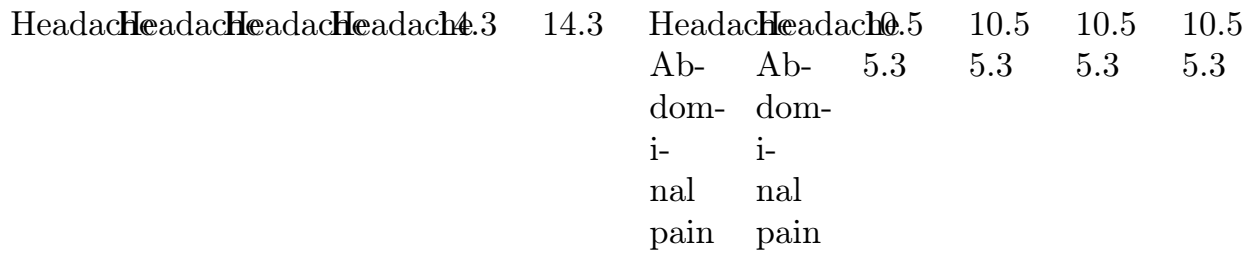

HampeLCTZ LCTZ LCTZ LCTZ LCTZ LCTZ Placebぬlaceb®laceb®laceb®lacebぬlacebo et $\quad\left(\begin{array}{lllll}1.25 \quad(1.25 \quad(1.25 \quad(1.25 \quad(1.25 \quad(1.25\end{array}\right.$

al., $\mathrm{mg} \quad \mathrm{mg} \mathrm{mg} \mathrm{mg} \mathrm{mg} \quad \mathrm{mg}$

2010 OD) OD) OD) OD) OD) OD)

(20) 


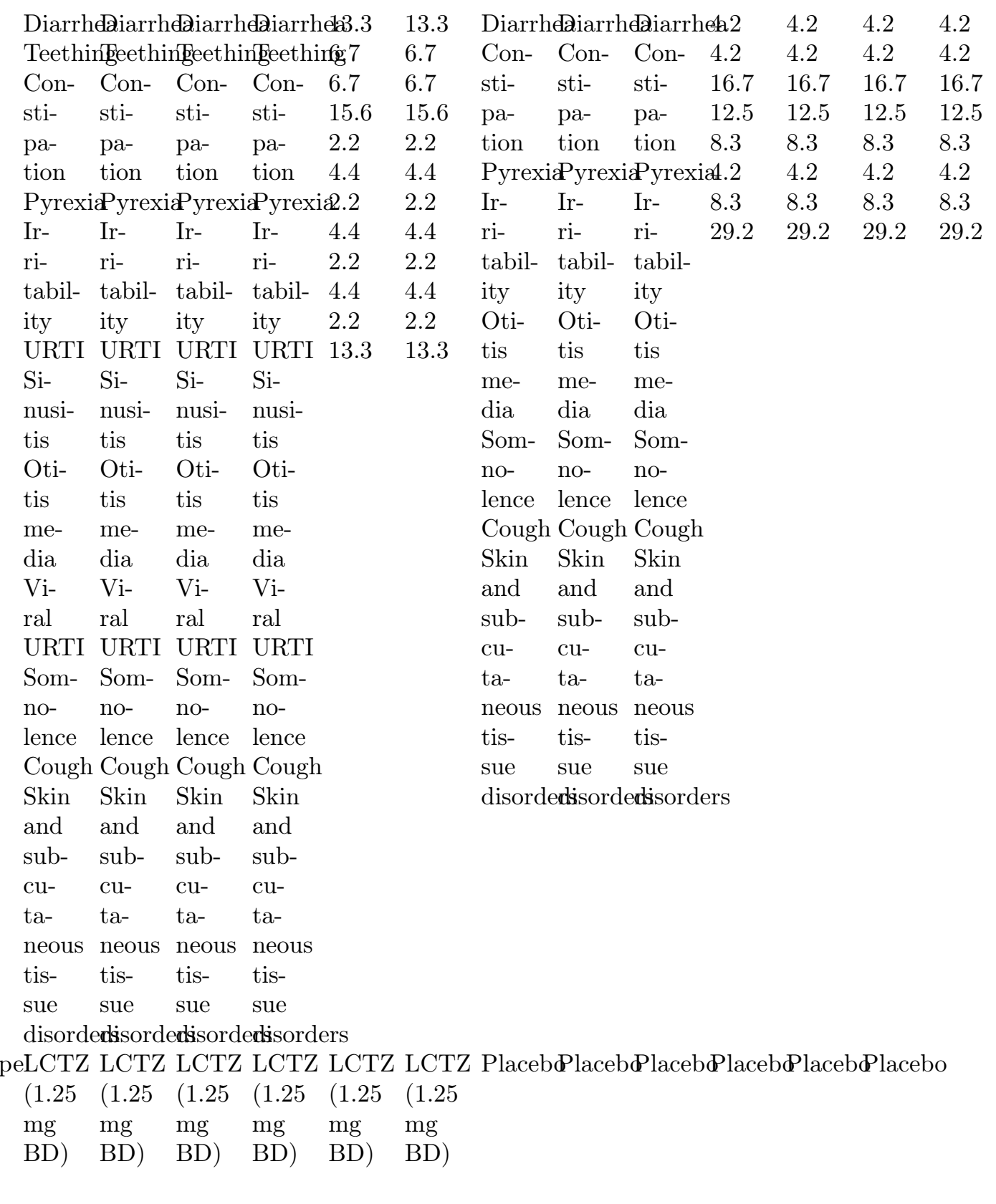




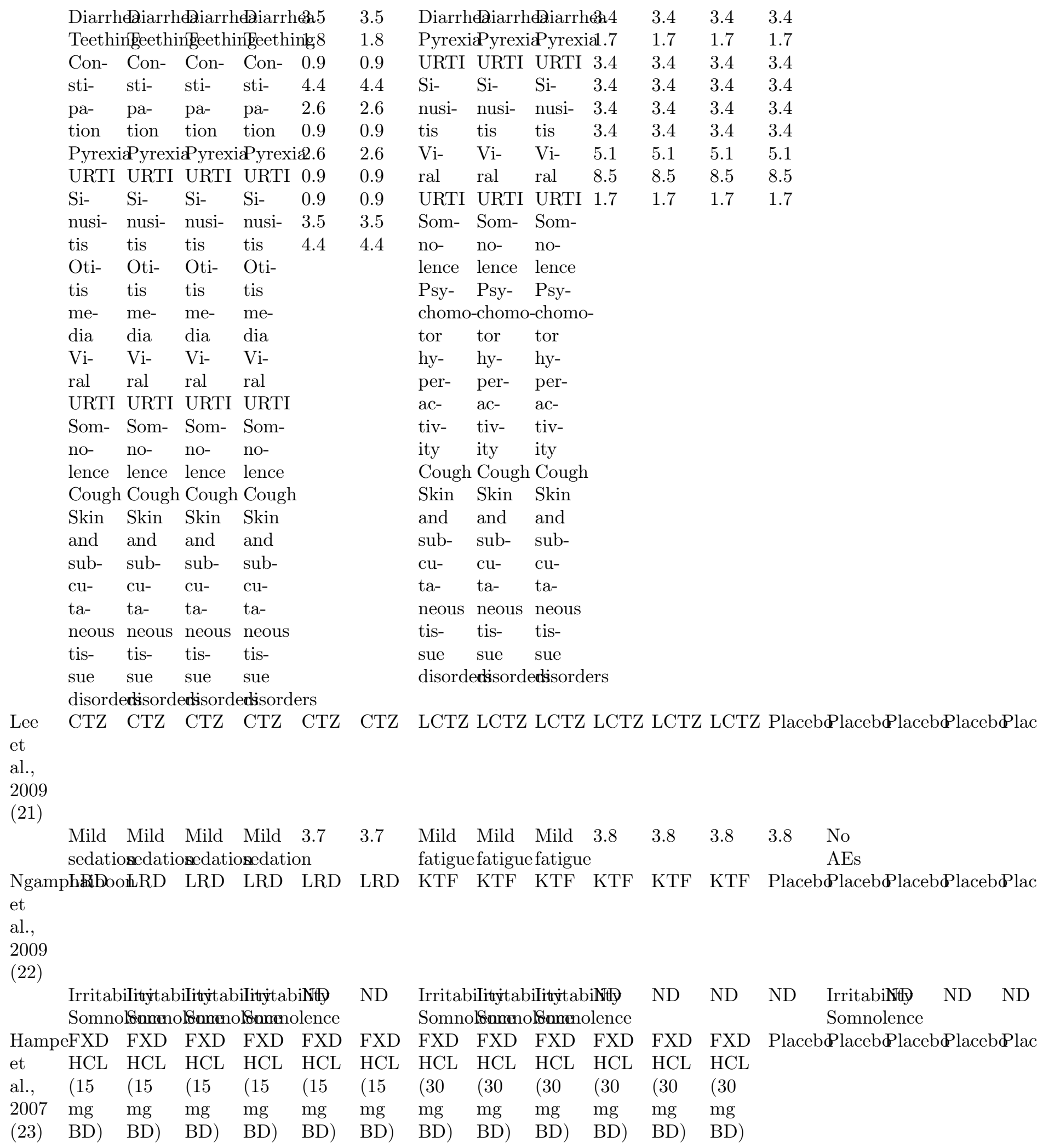




\begin{tabular}{|c|c|c|c|c|c|c|c|c|c|c|c|c|c|c|c|c|}
\hline \multicolumn{5}{|c|}{ 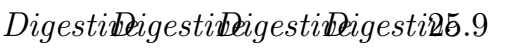 } & 25.9 & \multicolumn{4}{|c|}{ 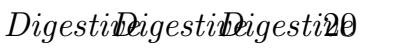 } & \multirow{2}{*}{$\begin{array}{l}20 \\
20\end{array}$} & \multirow{2}{*}{$\begin{array}{l}20 \\
20\end{array}$} & \multirow{2}{*}{$\begin{array}{l}20 \\
20\end{array}$} & \multicolumn{2}{|c|}{ Digestiæธ.9 } & \multirow{2}{*}{$\begin{array}{l}15.9 \\
14.5\end{array}$} & 15.9 \\
\hline sys- & sys- & sys- & sys- & 15.5 & 15.5 & sys- & sys- & sys- & 20 & & & & sys- & 14.5 & & 14.5 \\
\hline tem & tem & tem & tem & 5.2 & 5.2 & tem & tem & tem & 20 & 20 & 20 & 20 & tem & 1.4 & 1.4 & 1.4 \\
\hline Vom- & Vom- & Vom- & Vom- & 3.4 & 3.4 & Vom- & Vom- & Vom- & 20 & 20 & 20 & 20 & Vom- & 10.1 & 10.1 & 10.1 \\
\hline it- & it- & it- & it- & 12.2 & 12.2 & it- & it- & it- & & & & & it- & 5.8 & 5.8 & 5.8 \\
\hline ing & ing & ing & ing & 1.7 & 1.7 & ing & ing & ing & & & & & ing & 5.8 & 5.8 & 5.8 \\
\hline Di- & Di- & Di- & Di- & 1.7 & 1.7 & Res- & Res- & Res- & & & & & Di- & 1.4 & 1.4 & 1.4 \\
\hline ar- & ar- & ar- & ar- & 3.4 & 3.4 & $p i-$ & $p i-$ & $p i-$ & & & & & ar- & 2.9 & 2.9 & 2.9 \\
\hline rhea & rhea & rhea & rhea & 1.7 & 1.7 & $\mathrm{ra-}$ & $r a-$ & $r a-$ & & & & & rhea & 7.2 & 7.2 & 7.2 \\
\hline Gas- & Gas- & Gas- & Gas- & 3.4 & 3.4 & tory & tory & tory & & & & & Res- & 1.4 & 1.4 & 1.4 \\
\hline troen- & troen- & troen- & troen- & 1.7 & 1.7 & sys- & sys- & sys- & & & & & $p i-$ & & & \\
\hline teri- & teri- & teri- & teri- & 6.9 & 6.9 & tem & tem & tem & & & & & $r a-$ & & & \\
\hline & tis & tis & tis & & & URTI & URTI & URTI & & & & & tory & & & \\
\hline Res- & Res- & Res- & Res- & & & & & & & & & & sys- & & & \\
\hline$p i-$ & $p i-$ & $p i-$ & $p i-$ & & & & & & & & & & tem & & & \\
\hline $\mathrm{ra}-$ & $r a-$ & $r a-$ & $\mathrm{ra}-$ & & & & & & & & & & URTI & & & \\
\hline tory & tory & tory & tory & & & & & & & & & & Fever & & & \\
\hline sys- & sys- & sys- & sys- & & & & & & & & & & Un- & & & \\
\hline tem & tem & tem & tem & & & & & & & & & & in- & & & \\
\hline Cough & Cough & Cough & Cough & & & & & & & & & & ten- & & & \\
\hline & in- & in- & & & & & & & & & & & tional & & & \\
\hline creased & dcreased & dcreased & dcreased & & & & & & & & & & in- & & & \\
\hline URTI & URTI & URTI & URTI & & & & & & & & & & jury & & & \\
\hline Rhini- & Rhini- & Rhini- & Rhini- & & & & & & & & & & In- & & & \\
\hline & tis & tis & tis & & & & & & & & & & fec- & & & \\
\hline Fever & Fever & Fever & Fever & & & & & & & & & & tion & & & \\
\hline In- & In- & In- & In- & & & & & & & & & & Oti- & & & \\
\hline fec- & fec- & fec- & fec- & & & & & & & & & & tis & & & \\
\hline tion & tion & tion & tion & & & & & & & & & & me- & & & \\
\hline Oti- & Oti- & Oti- & Oti- & & & & & & & & & & dia & & & \\
\hline tis & tis & tis & tis & & & & & & & & & & Skin & & & \\
\hline me- & me- & me- & me- & & & & & & & & & & and & & & \\
\hline dia & dia & dia & dia & & & & & & & & & & $a p-$ & & & \\
\hline Skin & Skin & Skin & Skin & & & & & & & & & & penda & es & & \\
\hline and & and & and & and & & & & & & & & & & & & & \\
\hline$a p-$ & $a p-$ & $a p-$ & $a p-$ & & & & & & & & & & & & & \\
\hline pendag & gerendag & gqendag & gerendag & & & & & & & & & & & & & \\
\hline FXD & FXD & FXD & FXD & FXD & FXD & FXD & FXD & FXD & FXD & FXD & FXD & Pla & Placeb & Place & Place & \\
\hline HCL & HCL & HCL & HCL & HCL & HCL & HCL & HCL & HCL & HCL & HCL & HCL & & & & & \\
\hline 715 & $(15$ & $(15$ & $(15$ & $(15$ & $(15$ & $(30$ & $(30$ & $(30$ & $(30$ & $(30$ & $(30$ & & & & & \\
\hline mg & $\mathrm{mg}$ & $\mathrm{mg}$ & $\mathrm{mg}$ & $\mathrm{mg}$ & $\mathrm{mg}$ & $\mathrm{mg}$ & $\mathrm{mg}$ & $\mathrm{mg}$ & $\mathrm{mg}$ & $\mathrm{mg}$ & $\mathrm{mg}$ & & & & & \\
\hline BD) & $\mathrm{BD})$ & BD) & $\mathrm{BD})$ & $\mathrm{BD})$ & $\mathrm{BD})$ & BD) & $\mathrm{BD})$ & BD) & BD) & $\mathrm{BD})$ & $\mathrm{BD})$ & & & & & \\
\hline
\end{tabular}




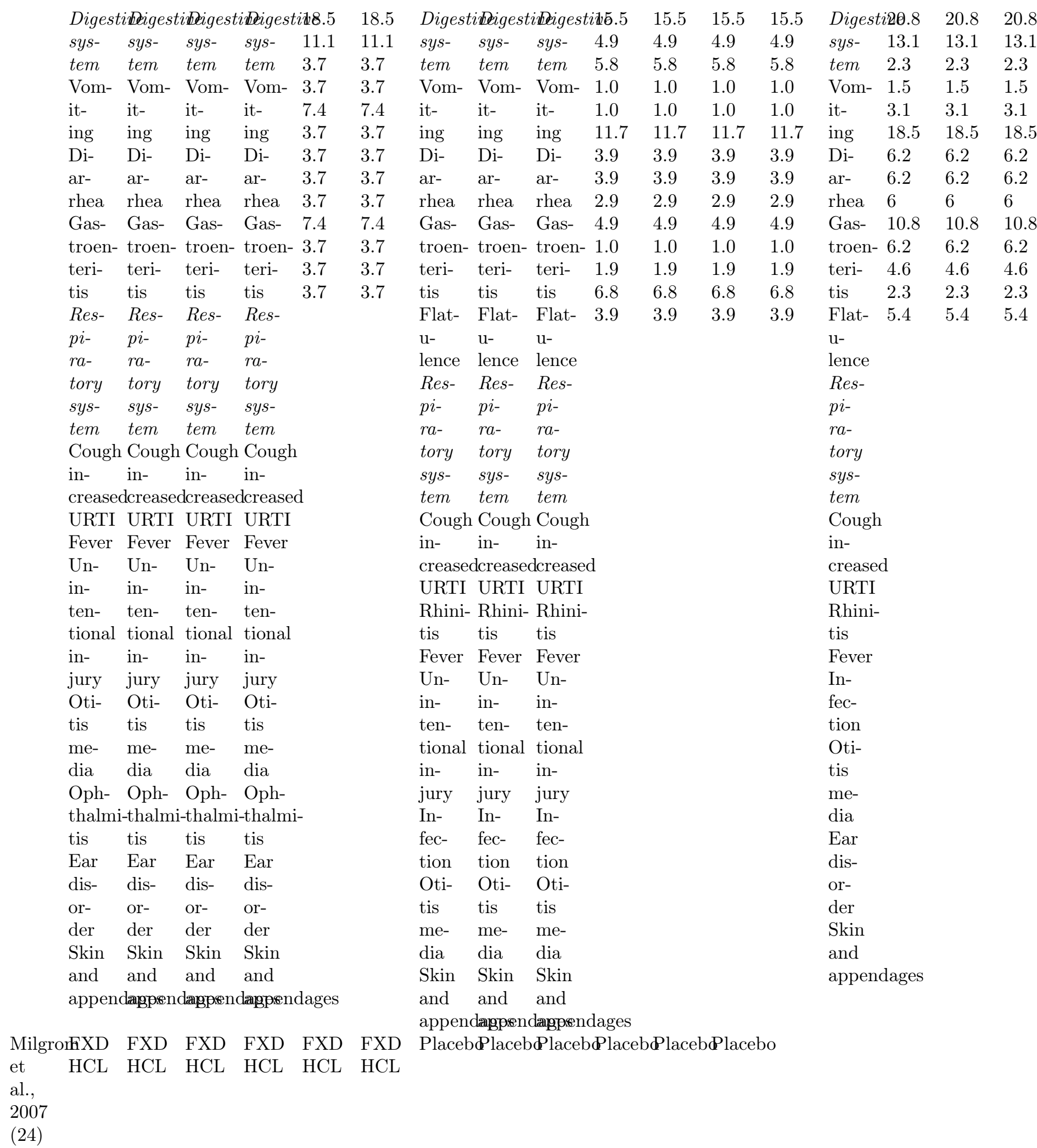




\begin{tabular}{|c|c|c|c|c|c|c|c|c|c|c|c|c|}
\hline Respirc & aARersyirc & äRensyirc & aARermir & 2013 & 20.3 & Respira & aRRersyira & aARersyir & altoray & 17.3 & 17.3 & 17.3 \\
\hline sys- & sys- & sys- & sys- & $4.5^{\circ}$ & 4.5 & sys- & sys- & sys- & 3.0 & 3.0 & 3.0 & 3.0 \\
\hline tem & tem & tem & tem & 4.1 & 4.1 & tem & tem & tem & 6.9 & 6.9 & 6.9 & 6.9 \\
\hline Cough & Cough & Cough & Cough & 4.1 & 4.1 & Cough & Cough & Cough & 2.6 & 2.6 & 2.6 & 2.6 \\
\hline in- & in- & in- & in- & 2.7 & 2.7 & in- & in- $\quad i$ & in- & 0.9 & 0.9 & 0.9 & 0.9 \\
\hline creased & tcreased & dcreased & dcreased & d5.9 & 5.9 & creased & dcreasedo & lcreasec & d5.6 & 5.6 & 5.6 & 5.6 \\
\hline URTI & URTI & URTI & URTI & 4.5 & 4.5 & URTI & URTI & URTI & 3.0 & 3.0 & 3.0 & 3.0 \\
\hline Rhini- & Rhini- & Rhini- & Rhini- & 3.6 & 3.6 & Rhini- & Rhini- I & Rhini- & 6.1 & 6.1 & 6.1 & 6.1 \\
\hline tis & tis & tis & tis & 5.0 & 5.0 & tis & tis $\quad t$ & tis & 4.8 & 4.8 & 4.8 & 4.8 \\
\hline Pharyn & Pharyn & $\mathrm{APharyn}$ & nPharyn & $\mathrm{n} 2.7$ & 2.7 & Pharyn & $\mathrm{nPharynl}$ & PPharyı & $n 2.6$ & 2.6 & 2.6 & 2.6 \\
\hline gi- & gi- & gi- & gi- & 2.3 & 2.3 & gi- & gi- & gi- & 3.0 & 3.0 & 3.0 & 3.0 \\
\hline tis & tis & tis & tis & 3.2 & 3.2 & tis & tis & tis & 3.9 & 3.9 & 3.9 & 3.9 \\
\hline Fever & Fever & Fever & Fever & 3.6 & 3.6 & Fever & Fever 1 & Fever & 1.3 & 1.3 & 1.3 & 1.3 \\
\hline Ac- & Ac- & Ac- & Ac- & 1.4 & 1.4 & Ac- & Ac- & Ac- & 2.6 & 2.6 & 2.6 & 2.6 \\
\hline $\begin{array}{l}\text { ci- } \\
\text { den- }\end{array}$ & $\begin{array}{l}\text { ci- } \\
\text { den- }\end{array}$ & $\begin{array}{l}\text { ci- } \\
\text { den- }\end{array}$ & $\begin{array}{l}\text { ci- } \\
\text { den- }\end{array}$ & 5.9 & 5.9 & $\begin{array}{l}\text { ci- } \\
\text { den- }\end{array}$ & $\begin{array}{l}\text { ci- } \\
\text { den- }\end{array}$ & $\begin{array}{l}\text { ci- } \\
\text { den- }\end{array}$ & 5.6 & 5.6 & 5.6 & 5.6 \\
\hline tal & tal & tal & tal & & & tal & tal & tal & & & & \\
\hline $\begin{array}{l}\text { in- } \\
\text { jury }\end{array}$ & $\begin{array}{l}\text { in- } \\
\text { jury }\end{array}$ & $\begin{array}{l}\text { in- } \\
\text { jury }\end{array}$ & $\begin{array}{l}\text { in- } \\
\text { jury }\end{array}$ & & & $\begin{array}{l}\text { in- } \\
\text { jury }\end{array}$ & $\begin{array}{l}\text { in- } \\
\text { jury }\end{array}$ & $\begin{array}{l}\text { in- } \\
\text { jury }\end{array}$ & & & & \\
\hline In- & In- & In- & In- & & & In- & In- & In- & & & & \\
\hline $\begin{array}{l}\text { fec- } \\
\text { tion }\end{array}$ & $\begin{array}{l}\text { fec- } \\
\text { tion }\end{array}$ & $\begin{array}{l}\text { fec- } \\
\text { tion }\end{array}$ & $\begin{array}{l}\text { fec- } \\
\text { tion }\end{array}$ & & & $\begin{array}{l}\text { fec- } \\
\text { tion }\end{array}$ & $\begin{array}{l}\text { fec- } \\
\text { tion }\end{array}$ & $\begin{array}{l}\text { fec- } \\
\text { tion }\end{array}$ & & & & \\
\hline Vom- & Vom- & Vom- & Vom- & & & Vom- & Vom- & Vom- & & & & \\
\hline it- & it- & it- & it- & & & it- & it- & it- & & & & \\
\hline ing & ing & ing & ing & & & ing & ing & ing & & & & \\
\hline GI & GI & GI & GI & & & GI & GI & GI & & & & \\
\hline pain & pain & pain & pain & & & pain & pain & pain & & & & \\
\hline Di- & Di- & Di- & Di- & & & Di- & Di- & Di- & & & & \\
\hline $\begin{array}{l}\text { ar- } \\
\text { rhea }\end{array}$ & $\begin{array}{l}\text { ar- } \\
\text { rhea }\end{array}$ & $\begin{array}{l}\text { ar- } \\
\text { rhea }\end{array}$ & $\begin{array}{l}\text { ar- } \\
\text { rhea }\end{array}$ & & & $\begin{array}{l}\text { ar- } \\
\text { rhea }\end{array}$ & $\begin{array}{l}\text { ar- } \\
\text { rhea }\end{array}$ & $\begin{array}{l}\text { ar- } \\
\text { rhea }\end{array}$ & & & & \\
\hline Headac & cheadac & cheadac & cheadac & che & & Headac & cheadacl & cheada & che & & & \\
\hline $\begin{array}{l}\text { Oti- } \\
\text { tis }\end{array}$ & $\begin{array}{l}\text { Oti- } \\
\text { tis }\end{array}$ & $\begin{array}{l}\text { Oti- } \\
\text { tis }\end{array}$ & $\begin{array}{l}\text { Oti- } \\
\text { tis }\end{array}$ & & & $\begin{array}{l}\text { Oti- } \\
\text { tis }\end{array}$ & $\begin{array}{l}\text { Oti- } \\
\text { tis }\end{array}$ & $\begin{array}{l}\text { Oti- } \\
\text { tis }\end{array}$ & & & & \\
\hline $\begin{array}{l}\text { me- } \\
\text { dia }\end{array}$ & $\begin{array}{l}\text { me- } \\
\text { dia }\end{array}$ & $\begin{array}{l}\text { me- } \\
\text { dia }\end{array}$ & $\begin{array}{l}\text { me- } \\
\text { dia }\end{array}$ & & & $\begin{array}{l}\text { me- } \\
\text { dia }\end{array}$ & $\begin{array}{l}\text { me- } \\
\text { dia }\end{array}$ & $\begin{array}{l}\text { me- } \\
\text { dia }\end{array}$ & & & & \\
\hline Ear & Ear & Ear & Ear & & & Ear & Ear & Ear & & & & \\
\hline pain & pain & pain & pain & & & pain & pain & pain & & & & \\
\hline $\begin{array}{l}\text { Spe- } \\
\text { cial }\end{array}$ & $\begin{array}{l}\text { Spe- } \\
\text { cial }\end{array}$ & $\begin{array}{l}\text { Spe- } \\
\text { cial }\end{array}$ & $\begin{array}{l}\text { Spe- } \\
\text { cial }\end{array}$ & & & $\begin{array}{l}\text { Spe- } \\
\text { cial }\end{array}$ & $\begin{array}{l}\text { Spe- } \\
\text { cial }\end{array}$ & $\begin{array}{l}\text { Spe- } \\
\text { cial }\end{array}$ & & & & \\
\hline senses & senses & senses & senses & & & senses & senses & senses & & & & \\
\hline
\end{tabular}

al.,2007

(25) 


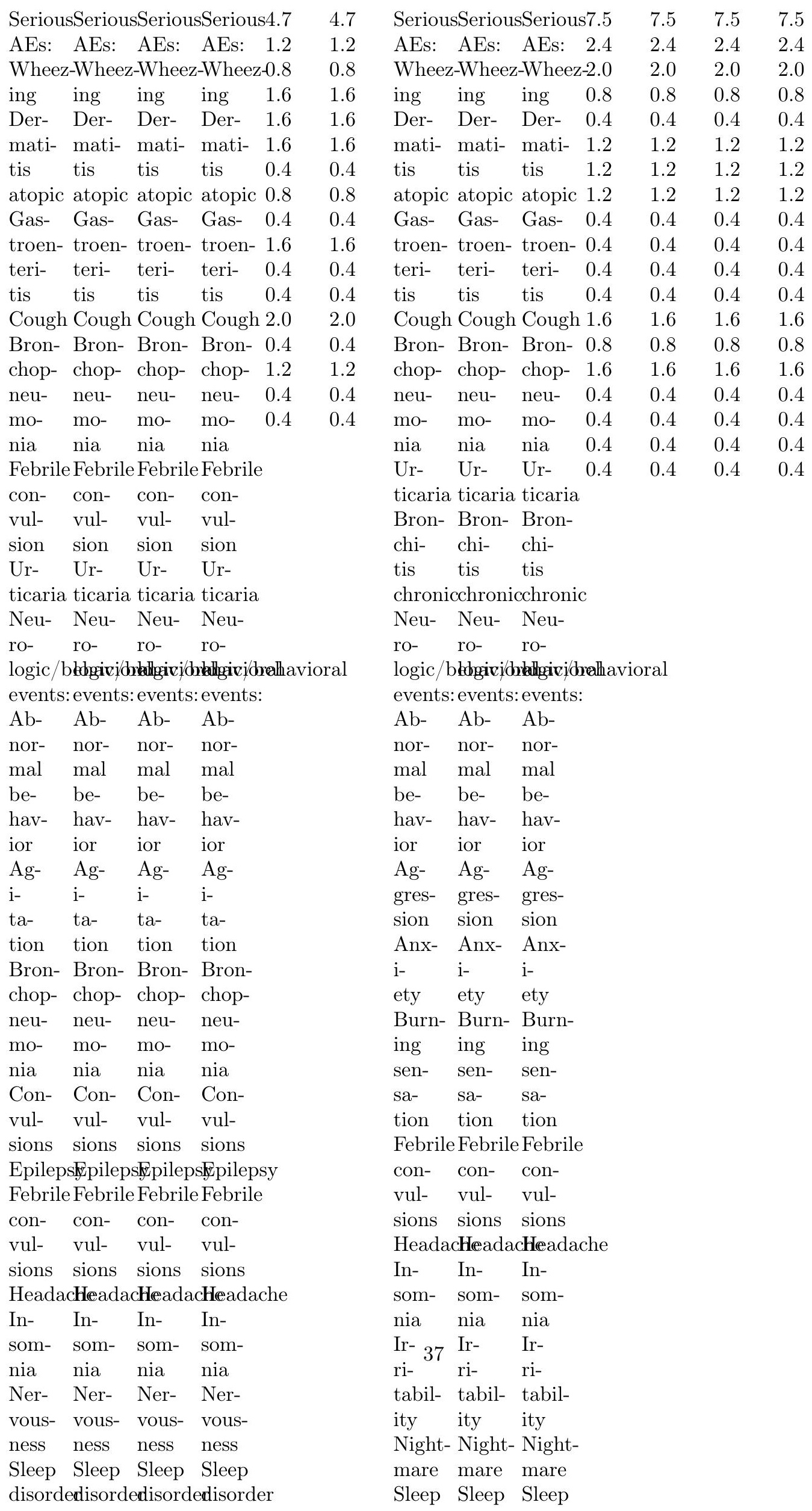


Chen CTZ CTZ CTZ CTZ CTZ CTZ MLK MLK MLK MLK MLK MLK PlacebPlaceb\&laceb\&laceb\&lac et al., 2006

(26)

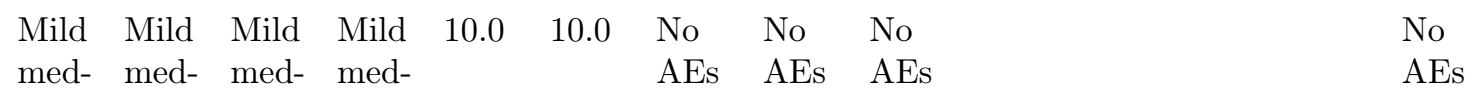

i- i- i- i-

ca- ca- ca- ca-

tion tion tion tion

in- in- in- in-

duced duced duced duced sedationedationedationedation

PrenneĐLRD DLRD DLRD DLRD DLRD DLRD Placeb\&laceb\&laceb\&laceb\&laceb\&lacebo

et

al.,

2006

(27) 


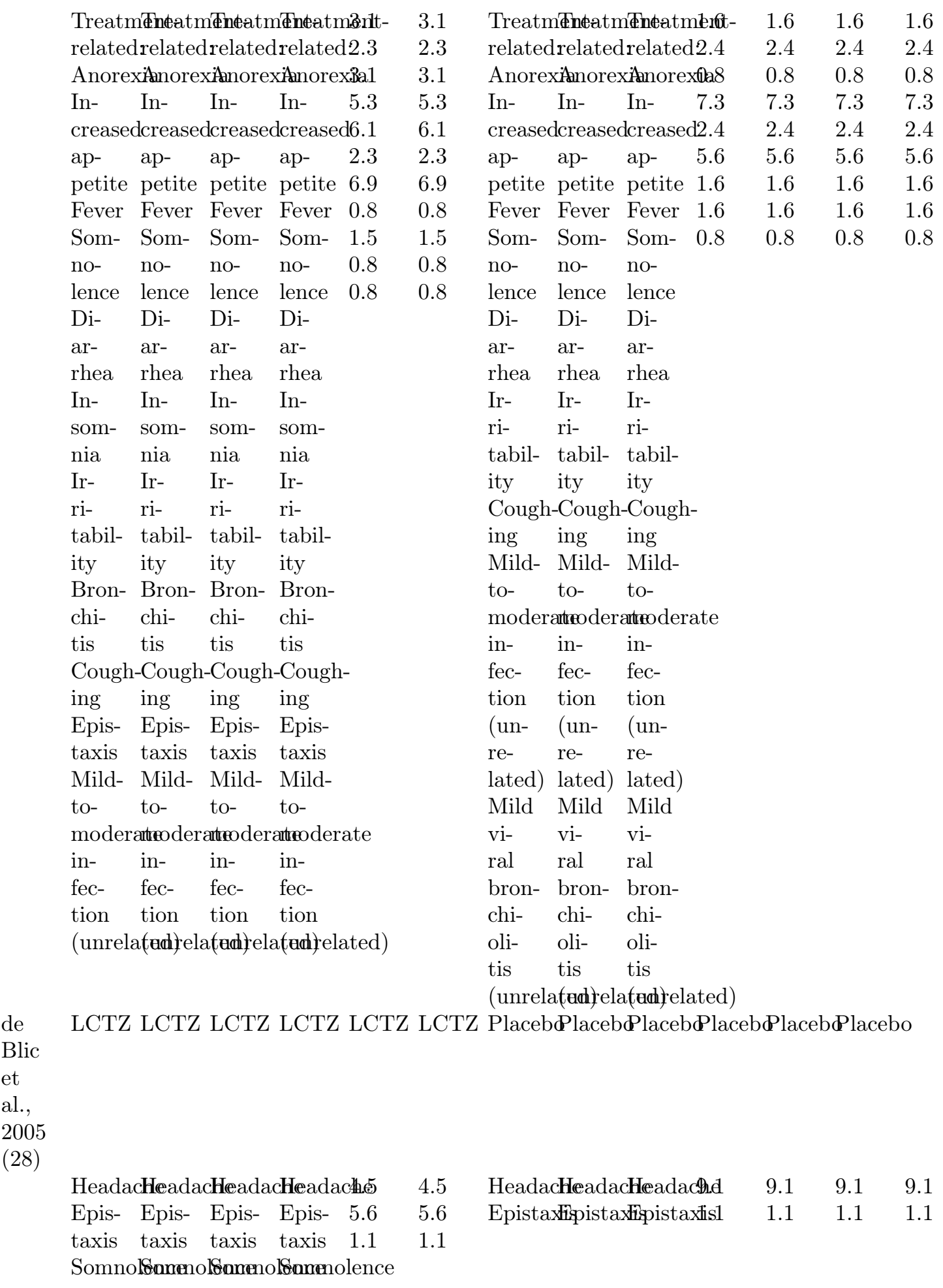


Potter LCTZ LCTZ LCTZ LCTZ LCTZ LCTZ Placeb®laceb®laceb®laceb®laceb®lacebo

et

al.,

2005

(29)

\begin{tabular}{|c|c|c|c|c|}
\hline Headacheadacheadacheadache.3 & 12.3 & Headacheadacheadache.2 & 11.2 & 11.2 \\
\hline URTI URTI URTI URTI & 8.4 & URTI URTI URTI 11.8 & 11.8 & 11.8 \\
\hline Influenðafluenðafluenđafluenø̌a2 & 5.2 & Influenđafluenđafluen9a2 & 9.2 & 9.2 \\
\hline
\end{tabular}

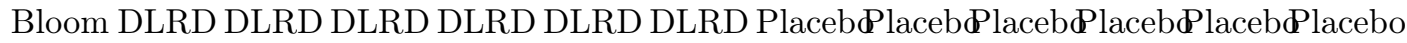

et

al.,

2004

$(30)$

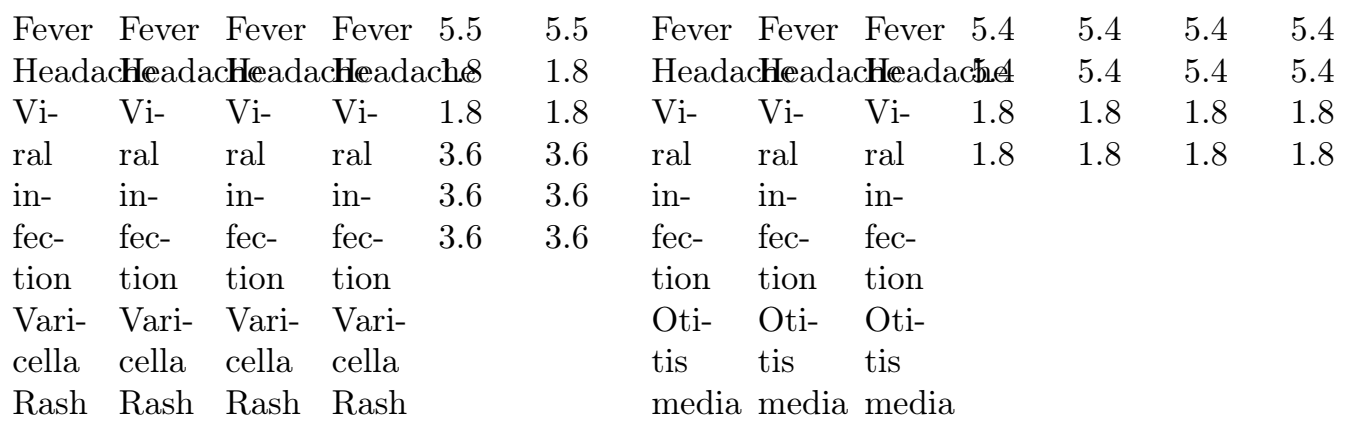

Uri- Uri- Uri- Uri-

nary nary nary nary

tract tract tract tract

infectioimfectiomfectioimfection

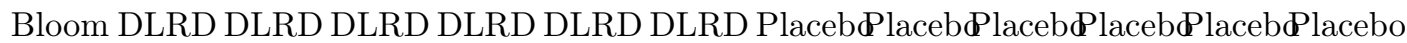

et

al.,

2004

$(30)$

$\begin{array}{llllll}\text { Headacheadacheadacheadache } \bar{\theta} & 1.7 & \text { Headacheadacheadactie } & 6.7 & 6.7 & 6.7\end{array}$

Gas- $\quad$ Gas- $\quad$ Gas- $\quad \begin{array}{llll}3.3 & 3.3 & 3.3 & 3.3\end{array}$

troen- troen- troen- $3.3 \quad 3.3 \quad 3.3 \quad 3.3$

teri- teri- teri-

tis tis tis

Vomitingomitingomiting

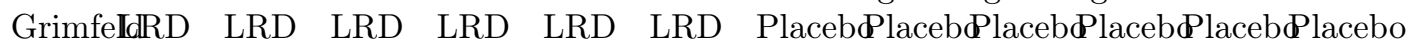

et

al.,

2004

$(31)$ 


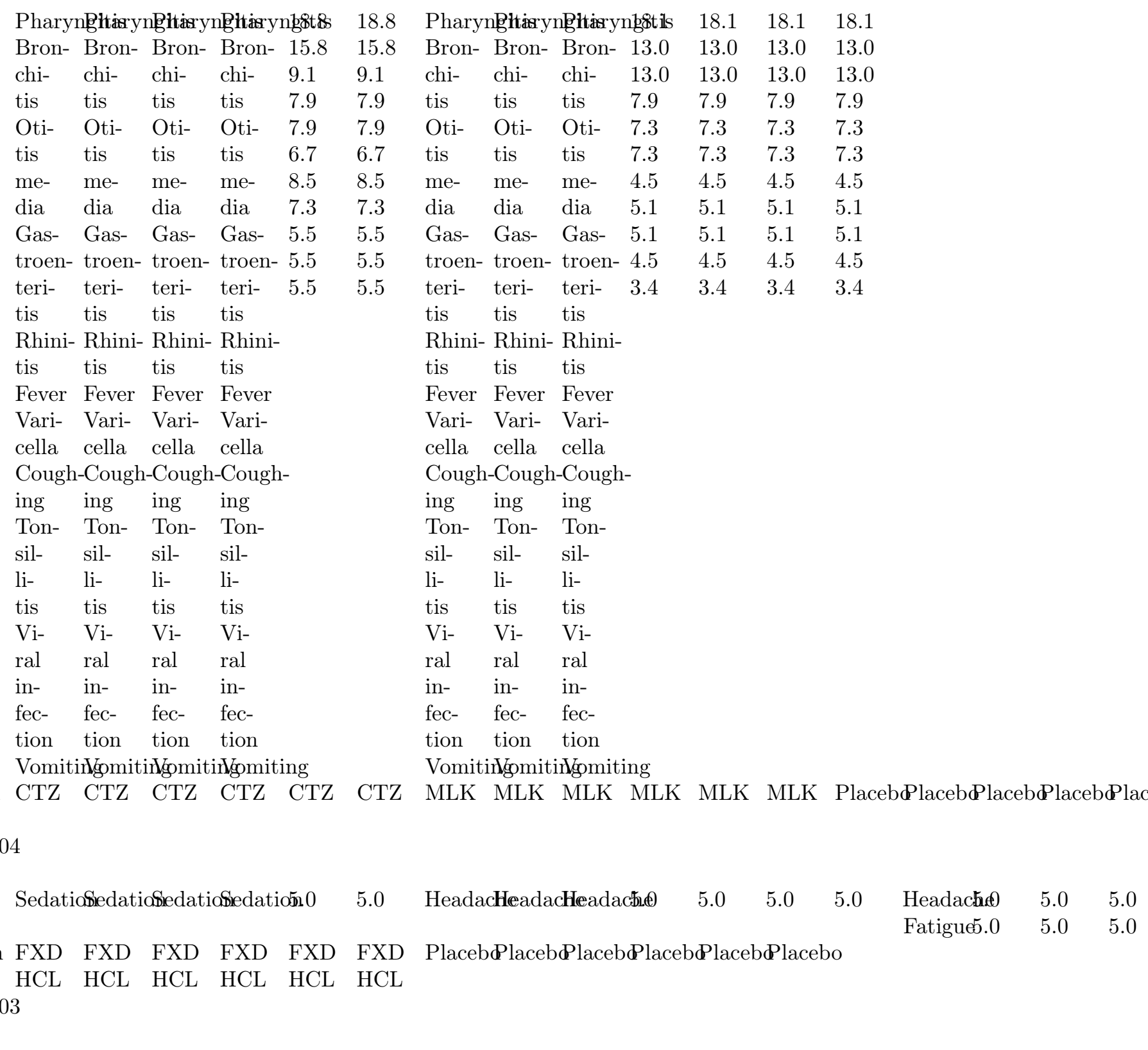




\begin{tabular}{|c|c|c|c|c|c|c|c|c|c|c|c|c|}
\hline \multicolumn{5}{|c|}{ Headacheadacheadacheadac刎® } & 5.0 & \multicolumn{4}{|c|}{ Headacheadacheadaches } & 2.8 & 2.8 & 2.8 \\
\hline Epis- & Epis- & Epis- & Epis- & 1.5 & 1.5 & Epis- & Epis- & Epis- & 1.1 & 1.1 & 1.1 & 1.1 \\
\hline taxis & taxis & taxis & taxis & 1.5 & 1.5 & taxis & taxis & taxis & 1.1 & 1.1 & 1.1 & 1.1 \\
\hline URTI & URTI & URTI & URTI & 1.3 & 1.3 & URTI & URTI & URTI & 0.2 & 0.2 & 0.2 & 0.2 \\
\hline Phary & nPharyı & nPharyn & nPharyn & $\mathrm{n} 7.3$ & 1.3 & Pharyn & nPhary & nPhary & $\mathrm{n} \theta .2$ & 0.2 & 0.2 & 0.2 \\
\hline gi- & gi- & gi- & gi- & 1.1 & 1.1 & gi- & gi- & gi- & 0.6 & 0.6 & 0.6 & 0.6 \\
\hline tis & tis & tis & tis & 1.1 & 1.1 & tis & tis & tis & 1.3 & 1.3 & 1.3 & 1.3 \\
\hline Si- & $\mathrm{Si}-$ & Si- & Si- & 0.9 & 0.9 & Nau- & Nau- & Nau- & 1.9 & 1.9 & 1.9 & 1.9 \\
\hline nusi- & nusi- & nusi- & nusi- & 0.6 & 0.6 & sea & sea & sea & 1.1 & 1.1 & 1.1 & 1.1 \\
\hline tis & tis & tis & tis & 0.2 & 0.2 & Rash & Rash & Rash & 1.1 & 1.1 & 1.1 & 1.1 \\
\hline Nau- & Nau- & Nau- & Nau- & 0.2 & 0.2 & Ac- & Ac- & Ac- & & & & \\
\hline & sea & sea & sea & 0.2 & 0.2 & & ci- & ci- & & & & \\
\hline Rash & Rash & Rash & Rash & & & den- & den- & den- & & & & \\
\hline & Ac- & Ac- & Ac- & & & & tal & tal & & & & \\
\hline & & & ci- & & & & in- & in- & & & & \\
\hline den- & den- & den- & den- & & & jury & jury & jury & & & & \\
\hline & tal & tal & tal & & & Asthm & 1asthm & nasthm & & & & \\
\hline & in- & in- & in- & & & In- & In- & In- & & & & \\
\hline jury & jury & jury & jury & & & fec- & fec- & fec- & & & & \\
\hline Asthm & lasthm & hasthm & nasthme & & & tion & tion & tion & & & & \\
\hline & In- & In- & In- & & & Gas- & Gas- & Gas- & & & & \\
\hline fec- & fec- & fec- & fec- & & & troin- & troin- & troin- & & & & \\
\hline tion & tion & tion & tion & & & testi- & testi- & testi- & & & & \\
\hline Gas- & Gas- & Gas- & Gas- & & & nal & nal & nal & & & & \\
\hline troin- & troin- & troin- & troin- & & & pain & pain & pain & & & & \\
\hline testi- & testi- & testi- & testi- & & & & & & & & & \\
\hline $\begin{array}{l}\text { nal } \\
\text { pain }\end{array}$ & $\begin{array}{l}\text { nal } \\
\text { pain }\end{array}$ & $\begin{array}{l}\text { nal } \\
\text { pain }\end{array}$ & $\begin{array}{l}\text { nal } \\
\text { pain }\end{array}$ & & & & & & & & & \\
\hline Neu- & Neu- & Neu- & Neu- & & & & & & & & & \\
\hline trope- & trope- & trope- & trope- & & & & & & & & & \\
\hline nia & nia & nia & nia & & & & & & & & & \\
\hline (sever & e) severe & e) severe & e) & & & & & & & & & \\
\hline CTZ & CTZ & CTZ & CTZ & CTZ & CTZ & Placeb & \&laceb & bळlacel & bळla & b®las & bळlaceb & \\
\hline
\end{tabular}

(34) 


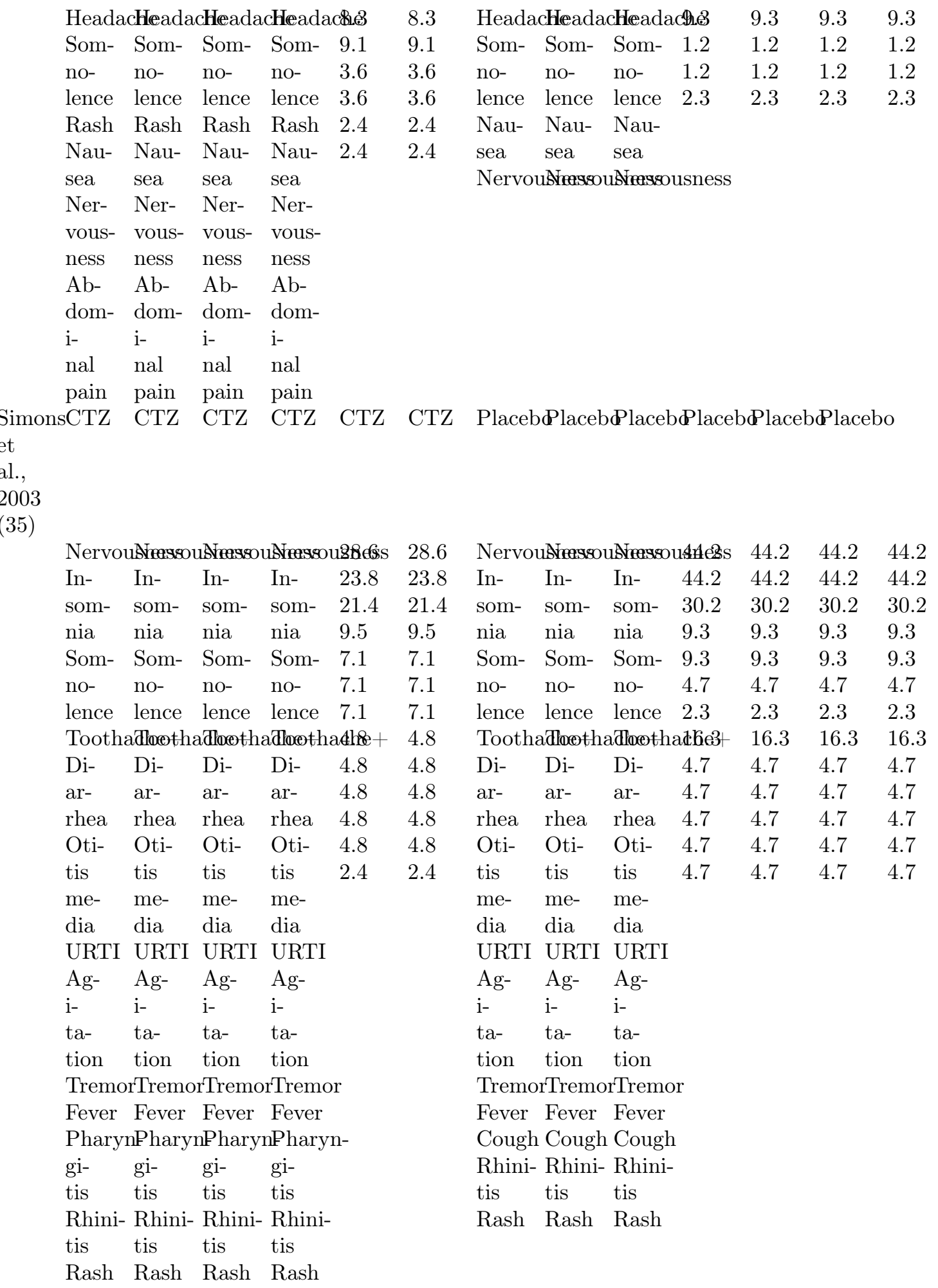




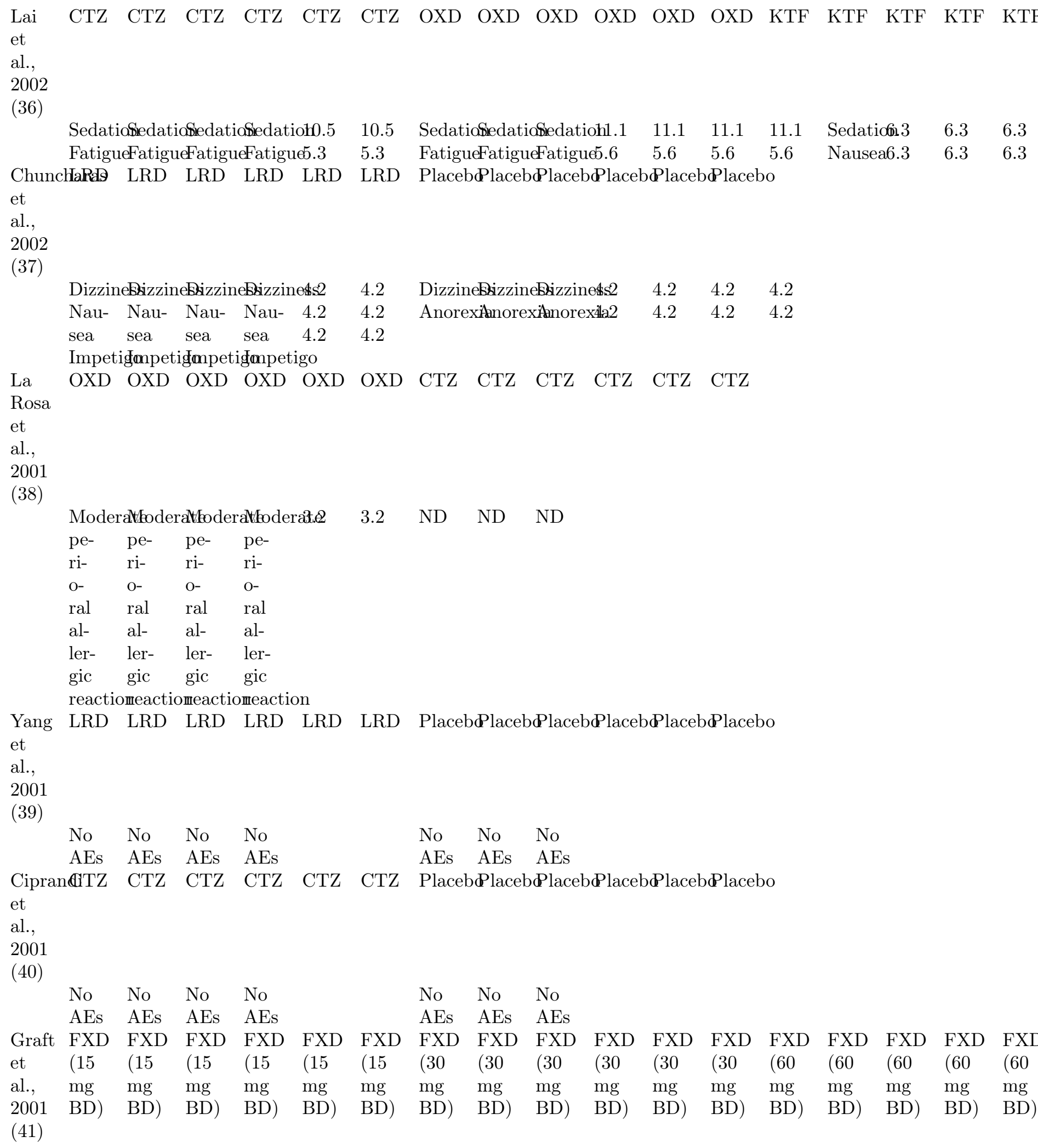




\begin{tabular}{|c|c|c|c|c|c|c|c|c|c|c|c|c|c|c|c|}
\hline URTI & URTI & URTI & URTI & 4.9 & 4.9 & URTI & URTI & URTI & 4.3 & 4.3 & 4.3 & 4.3 & URTI 1.4 & 1.4 & 1.4 \\
\hline Phary & nPharyr & nPharyı & nPhary & $\mathrm{n} 4.0$ & 4.0 & Phary & nPharyr & nPharyr & $\mathrm{n} 2.9$ & 2.9 & 2.9 & 2.9 & Pharynz.8 & 2.8 & 2.8 \\
\hline gi- & gi- & gi- & gi- & 1.3 & 1.3 & gi- & gi- & gi- & 3.8 & 3.8 & 3.8 & 3.8 & gi- $\quad 2.3$ & 2.3 & 2.3 \\
\hline tis & tis & tis & tis & 1.8 & 1.8 & tis & tis & tis & 2.9 & 2.9 & 2.9 & 2.9 & 4.2 & 4.2 & 4.2 \\
\hline Cough & -Cough & 1-Cough & -Cough & $1-2.7$ & 2.7 & Cough & -Cough & -Cough & -1.9 & 1.9 & 1.9 & 1.9 & Cough-2.3 & 2.3 & 2.3 \\
\hline ing & ing & ing & ing & 1.8 & 1.8 & ing & ing & ing & 2.4 & 2.4 & 2.4 & 2.4 & ing $\quad 1.9$ & 1.9 & 1.9 \\
\hline In- & In- & In- & In- & 8.0 & 8.0 & In- & In- & In- & 7.2 & 7.2 & 7.2 & 7.2 & In- & 9.4 & 9.4 \\
\hline jury & jury & jury & jury & & & jury & jury & jury & 0.5 & 0.5 & 0.5 & 0.5 & jury & & \\
\hline ac- & ac- & ac- & $\mathrm{ac}-$ & & & ac- & ac- & ac- & & & & & ac- & & \\
\hline $\begin{array}{l}\text { ci- } \\
\text { dent }\end{array}$ & $\begin{array}{l}\text { ci- } \\
\text { dent }\end{array}$ & $\begin{array}{l}\text { ci- } \\
\text { dent }\end{array}$ & $\begin{array}{l}\text { ci- } \\
\text { dent }\end{array}$ & & & $\begin{array}{l}\text { ci- } \\
\text { dent }\end{array}$ & $\begin{array}{l}\text { ci- } \\
\text { dent }\end{array}$ & $\begin{array}{l}\text { ci- } \\
\text { dent }\end{array}$ & & & & & $\begin{array}{l}\text { ci- } \\
\text { dent }\end{array}$ & & \\
\hline $\mathrm{Ab}-$ & $\mathrm{Ab}-$ & $\mathrm{Ab}-$ & $\mathrm{Ab}-$ & & & $\mathrm{Ab}-$ & $\mathrm{Ab}-$ & $\mathrm{Ab}-$ & & & & & $\mathrm{Ab}-$ & & \\
\hline dom- & dom- & dom- & dom- & & & dom- & dom- & dom- & & & & & dom- & & \\
\hline & i- & & i- & & & & & i- & & & & & i- & & \\
\hline nal & nal & nal & nal & & & nal & nal & nal & & & & & nal & & \\
\hline pain & pain & pain & pain & & & pain & pain & pain & & & & & pain & & \\
\hline Fever & Fever & Fever & Fever & & & Fever & Fever & Fever & & & & & Fever & & \\
\hline Heada & cheadac & cheada & cheada & che & & Heada & cheadac & cheadac & che & & & & Headache & & \\
\hline
\end{tabular}

SalmunLRD LRD LRD LRD LRD LRD Placebهlaceb®laceb\&laceb®lacebهlacebo et al., 2000 $(42)$

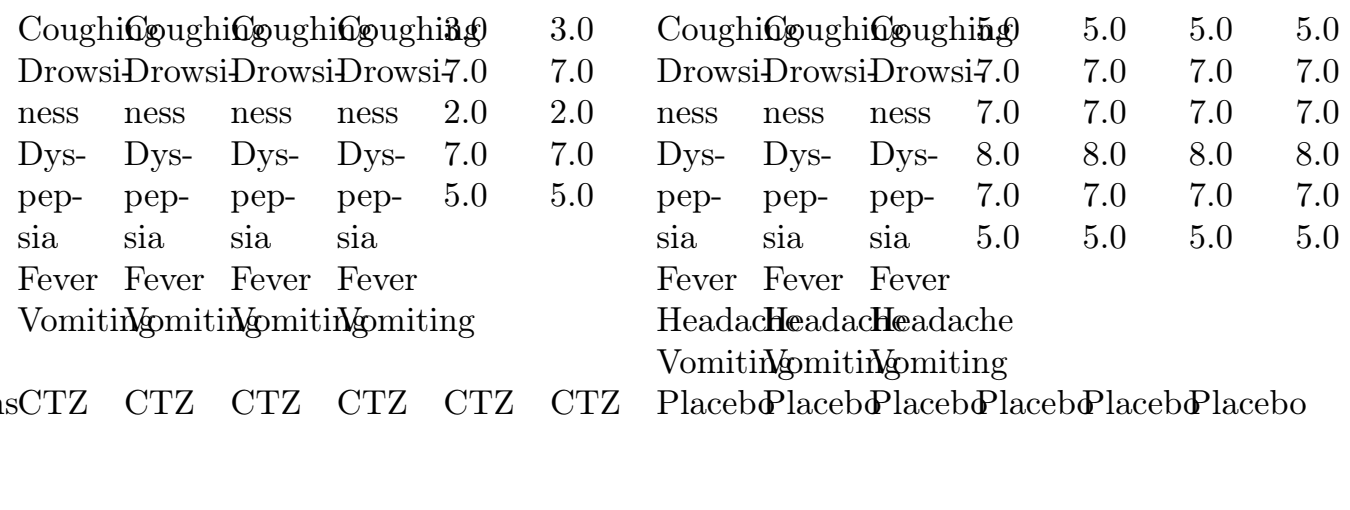
1999 


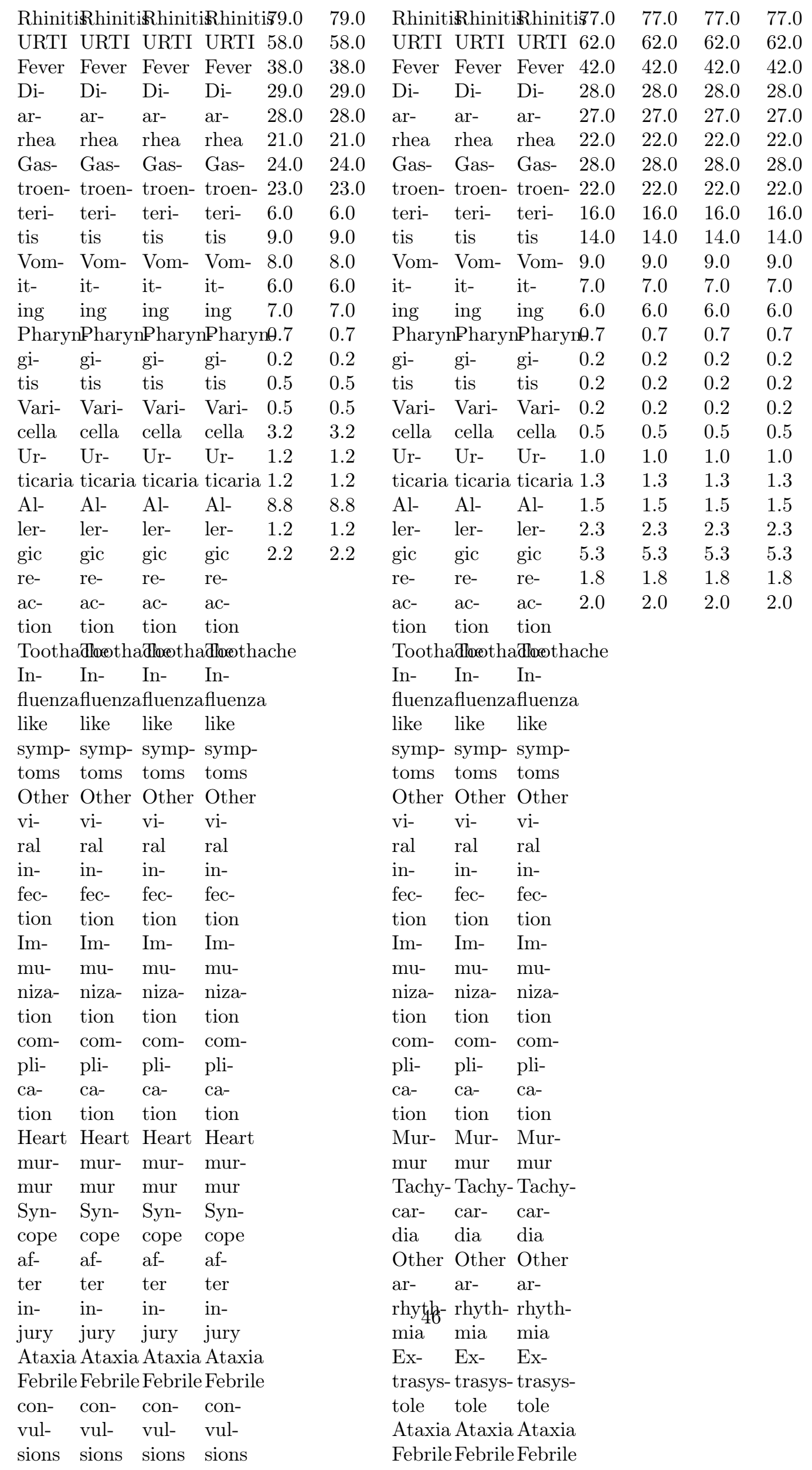


Wahn CTZ CTZ CTZ CTZ CTZ CTZ Placeb\&laceb\&lacebdlacebPlaceb\&lacebo et al., 1998

$(44)$ 


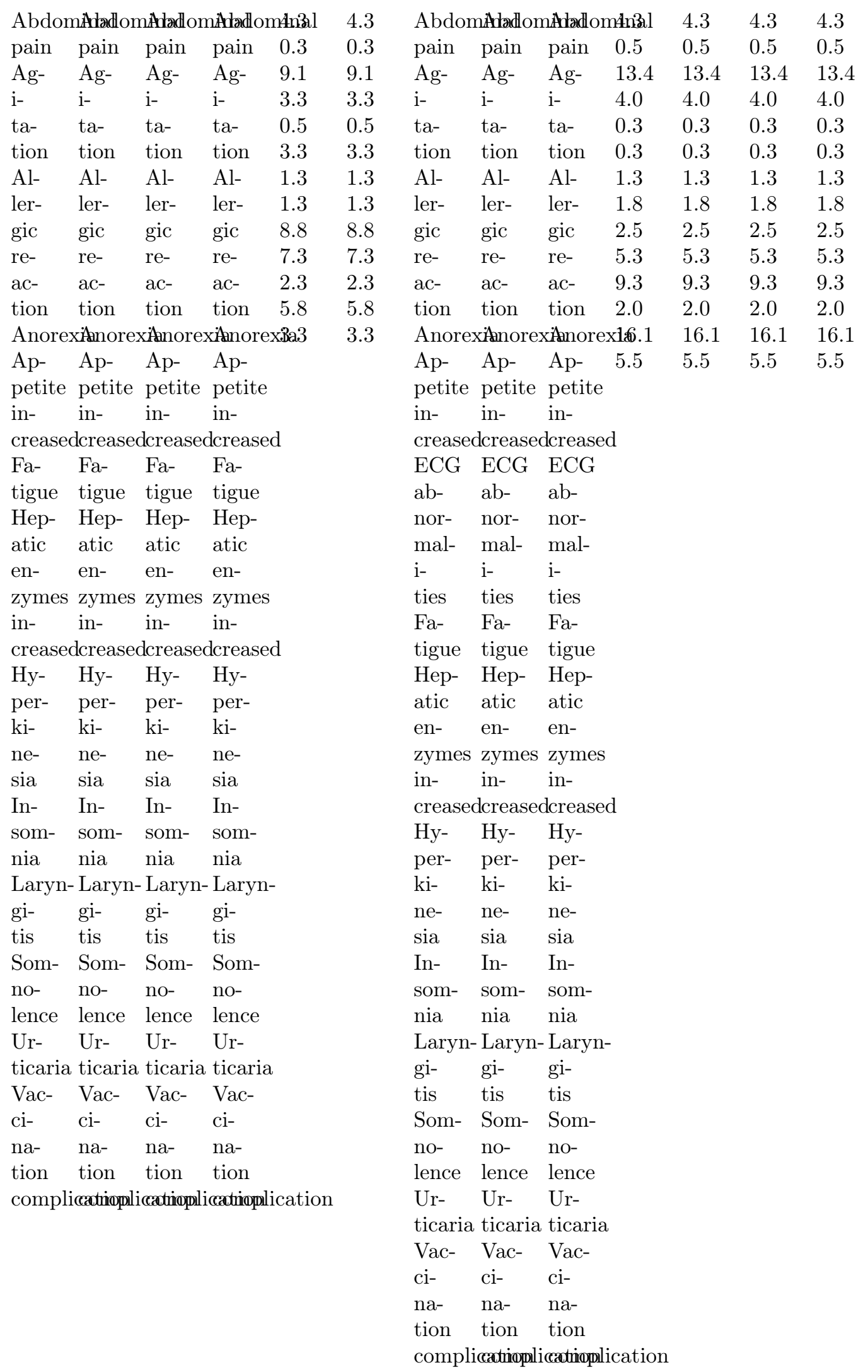


Sienra-CTZ CTZ CTZ CTZ CTZ CTZ LRD LRD LRD LRD LRD LRD

Monge

et

al.,

1999

(45)

Somnoldamoldamonoldamoler.jece 2.5 No No No

and and and and 2.5 2.5 AEs AEs AEs

mild mild mild mild

ir- ir- ir- ir-

ri- ri- ri- ri-

tabil- tabil- tabil- tabil-

ity ity ity ity

Gen- Gen- Gen- Gen-

er- er- er- er-

al- al- al- al-

ized ized ized ized

rash rash rash rash

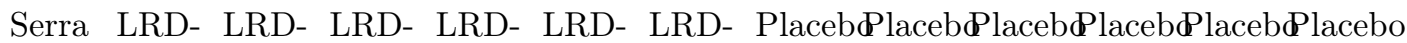

et PSD. PSD. PSD. PSD. PSD. PSD.

al.,

1998

(46)

Slight Slight Slight Slight 5.0 5.0 No No No

tran- tran- tran- tran-

AEs AEs AEs

sient sient sient sient

insomniasomniasomniasomnia

DelgadळRD LRD LRD LRD LRD LRD CTZ CTZ CTZ

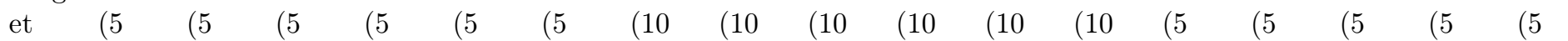

al., $\mathrm{mg} \quad \mathrm{mg} \mathrm{mg} \mathrm{mg} \quad \mathrm{mg} \quad \mathrm{mg} \quad \mathrm{mg} \quad \mathrm{mg} \quad \mathrm{mg} \quad \mathrm{mg} \quad \mathrm{mg} \quad \mathrm{mg} \quad \mathrm{mg} \quad \mathrm{mg} \quad \mathrm{mg} \quad \mathrm{mg} \quad \mathrm{mg}$

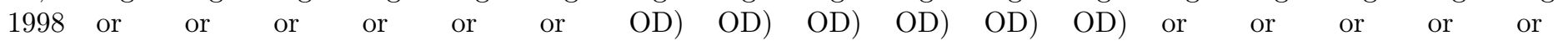

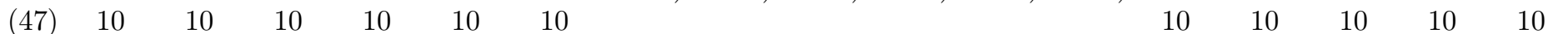

$\mathrm{mg} \quad \mathrm{mg} \quad \mathrm{mg} \quad \mathrm{mg} \quad \mathrm{mg} \quad \mathrm{mg} \quad$ mg $\mathrm{mg} \quad \mathrm{mg} \quad \mathrm{mg} \quad \mathrm{mg}$

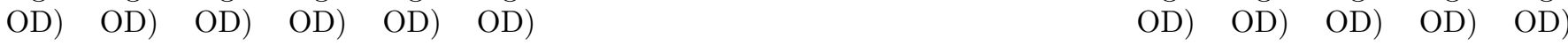

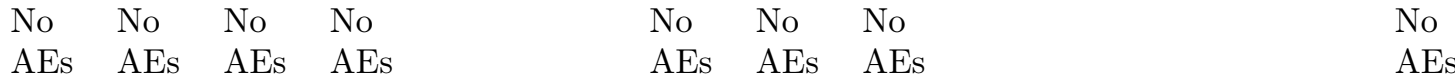

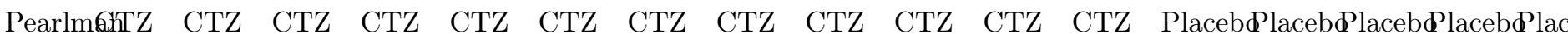

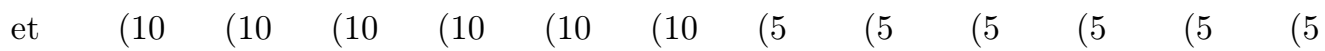

al.,1997mg $\mathrm{mg} \quad \mathrm{mg} \quad \mathrm{mg} \quad \mathrm{mg} \quad \mathrm{mg} \quad \mathrm{mg} \quad \mathrm{mg} \quad \mathrm{mg} \quad \mathrm{mg} \quad \mathrm{mg} \quad \mathrm{mg}$

$\left(\begin{array}{lllllllllllll}48 & \mathrm{OD}) & \mathrm{OD}) & \mathrm{OD}) & \mathrm{OD}) & \mathrm{OD}) & \mathrm{OD}) & \mathrm{OD}) & \mathrm{OD}) & \mathrm{OD}) & \mathrm{OD}) & \mathrm{OD} & \mathrm{OD}\end{array}\right.$

and

Winder

et

al.,1996

(49) 


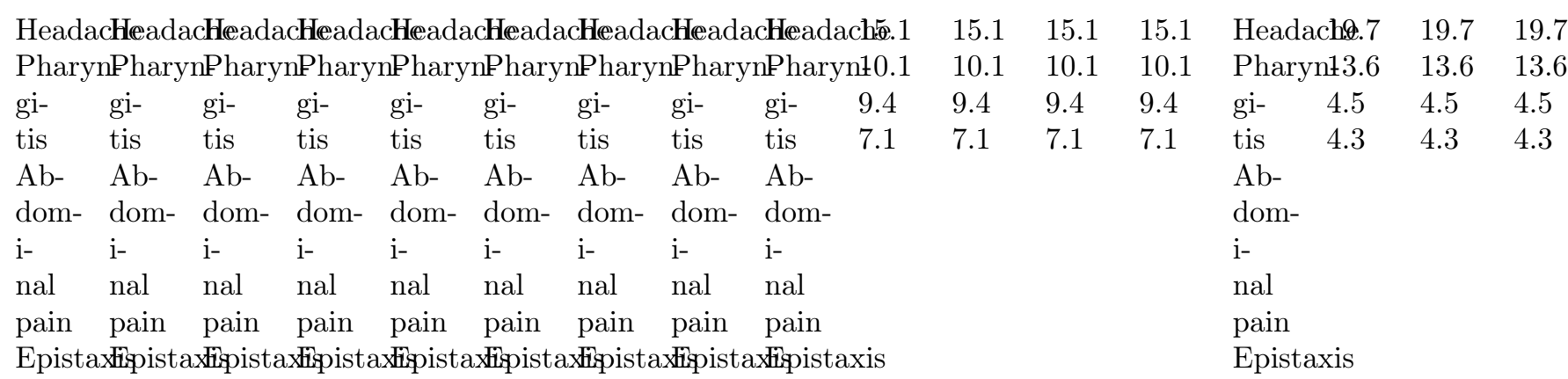

Cipran\&TZ CTZ CTZ CTZ CTZ CTZ Placeb\&laceb®lacebぬlaceb®laceb\&lacebo

et

al.,

1997

(50)

Headacheadacheadacheadach@.0 $\quad 10.0 \quad$ HeadacheadacheadacBe.0 $20.0 \quad 20.0 \quad 20.0$

BenedietisZ CTZ CTZ CTZ CTZ CTZ OXD OXD OXD OXD OXD OXD

et

al.,

1997

(51)

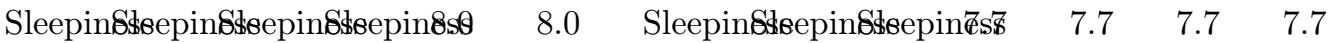

SlightlySlightlySlightly1.9 $\quad 1.9 \quad 1.9 \quad 1.9$

in- in- in-

creasedcreasedcreased

SGPT SGPT SGPT

Tinkelnáñ CTZ CTZ CTZ CTZ CTZ CTZ CTZ CTZ CTZ CTZ CTZ CPN CPN CPN CPN CPN

et $\quad(5-\quad(5-\quad(5-\quad(5-\quad) \quad(5-\quad) \quad(5-\quad) \quad(2.5-\quad) \quad(2.5-\quad) \quad(2.5-\quad) \quad(2.5-\quad) \quad(2.5-\quad) \quad(2.5-$

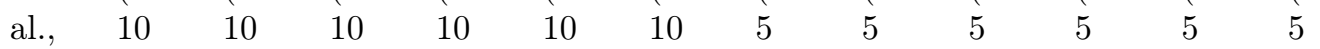

$1996 \mathrm{mg} \quad \mathrm{mg} \quad \mathrm{mg} \quad \mathrm{mg} \quad \mathrm{mg} \quad \mathrm{mg} \quad \mathrm{mg} \quad \mathrm{mg} \quad \mathrm{mg} \quad \mathrm{mg} \quad \mathrm{mg} \quad \mathrm{mg}$

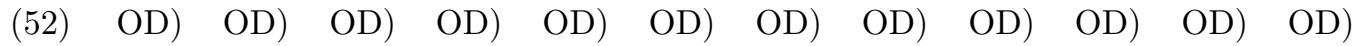

Abdomtlom

pain

Som- Som- Som- Som- Som- Som- Som- Som- Som- 4.0 4.0 4.04 .0

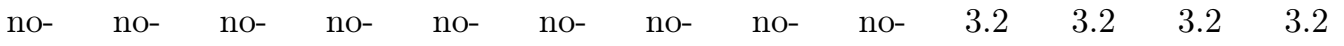

lence lence lence lence lence lence lence lence lence

Fa- Fa- Fa- Fa- Fa- Fa- Fa- Fa- Fa-

tigue tigue tigue tigue tigue tigue tigue tigue tigue

Nau- Nau- Nau- Nau- Nau- Nau- Nau- Nau- Nau-

sea sea sea sea sea sea sea sea sea

and and and and and and and and and

headactheadacheadacheadachneadactheadacheadacheadacheadache

Fasce CTZ CTZ CTZ CTZ CTZ CTZ Placeb\&laceb\&lacebdlacebPlaceb\&lacebo

et

al.,1996

(53)

ND ND ND ND

ND ND ND 


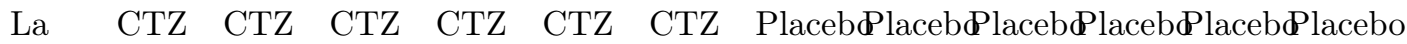

Rosa

et

al., 1994

(54)

\begin{tabular}{|c|c|c|c|c|c|c|c|c|c|c|c|c|c|c|c|c|c|}
\hline & No & No & No & No & & & ND & ND & ND & & & & & & & & \\
\hline & AEs & AEs & AEs & AEs & & & & & & & & & & & & & \\
\hline $\begin{array}{l}\text { Jobst } \\
\text { et }\end{array}$ & $\begin{array}{l}\text { CTZ } \\
(2.5\end{array}$ & $\begin{array}{l}\text { CTZ } \\
(2.5\end{array}$ & $\begin{array}{l}\text { CTZ } \\
(2.5\end{array}$ & $\begin{array}{l}\text { CTZ } \\
(2.5\end{array}$ & $\begin{array}{l}\text { CTZ } \\
(2.5\end{array}$ & $\begin{array}{l}\text { CTZ } \\
(2.5\end{array}$ & $\begin{array}{l}\mathrm{CTZ} \\
(5\end{array}$ & $\begin{array}{l}\mathrm{CTZ} \\
(5\end{array}$ & $\begin{array}{l}\text { CTZ } \\
(5\end{array}$ & $\begin{array}{l}\text { CTZ } \\
(5\end{array}$ & $\begin{array}{l}\text { CTZ } \\
(5\end{array}$ & $\begin{array}{l}\text { CTZ } \\
(5\end{array}$ & $\begin{array}{l}\text { CTZ } \\
(10\end{array}$ & $\begin{array}{l}\text { CTZ } \\
(10\end{array}$ & $\begin{array}{l}\text { CTZ } \\
(10\end{array}$ & $\begin{array}{l}\text { CTZ } \\
(10\end{array}$ & $\begin{array}{l}\mathrm{CT} \\
(10\end{array}$ \\
\hline al.,199 & $4 \mathrm{mg}$ & $\mathrm{mg}$ & mg & mg & $\mathrm{mg}$ & mg & $\mathrm{mg}$ & $\mathrm{mg}$ & mg & mg & mg & mg & mg & $\mathrm{mg}$ & mg & mg & $\mathrm{mg}$ \\
\hline & ND & ND & ND & ND & & & ND & ND & ND & & & & & ND & & & \\
\hline
\end{tabular}

AllegraCTZ CTZ CTZ CTZ CTZ CTZ Placebهlaceb\&lacebPlacebهlacebهlacebo et al.,1993

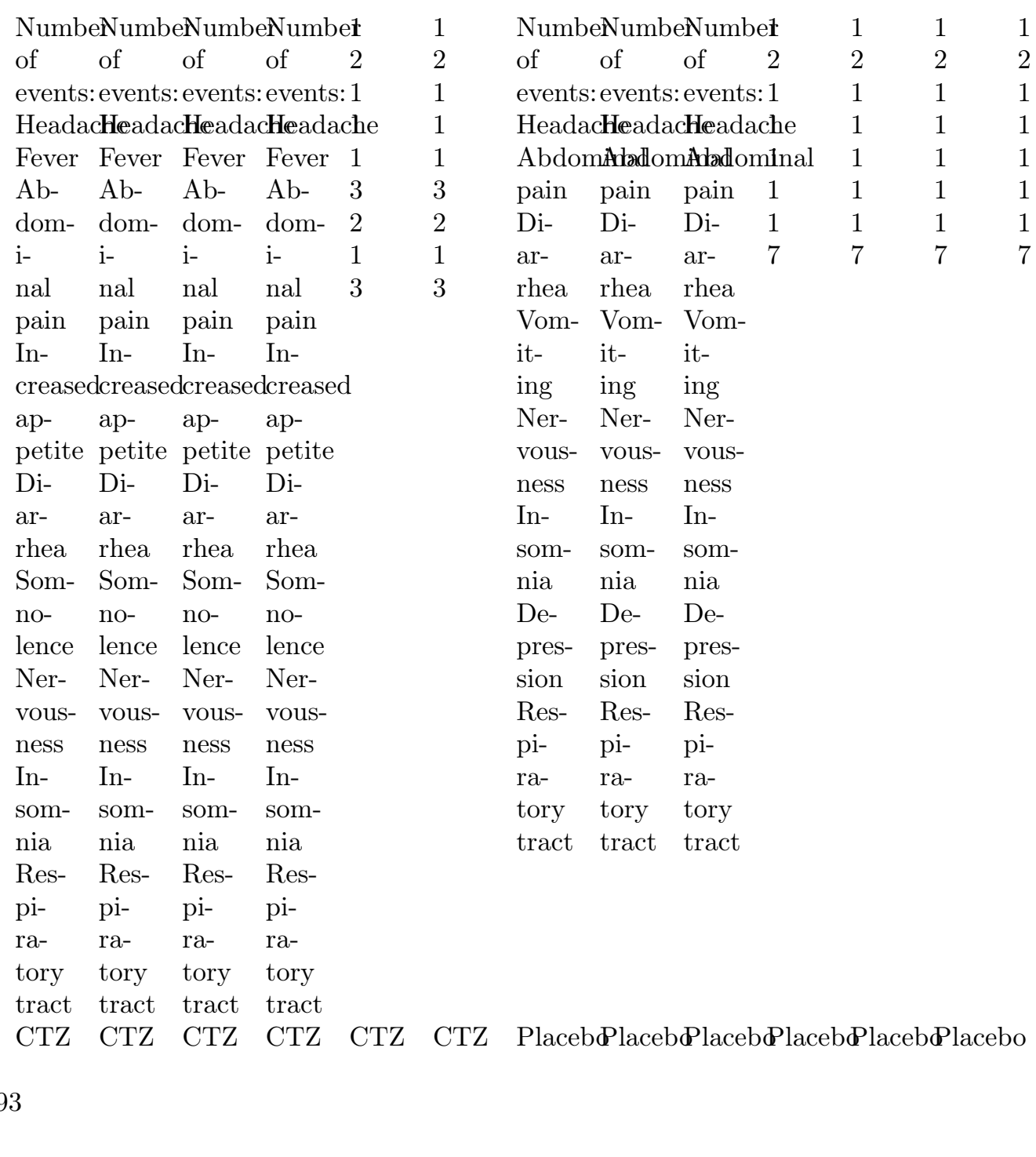




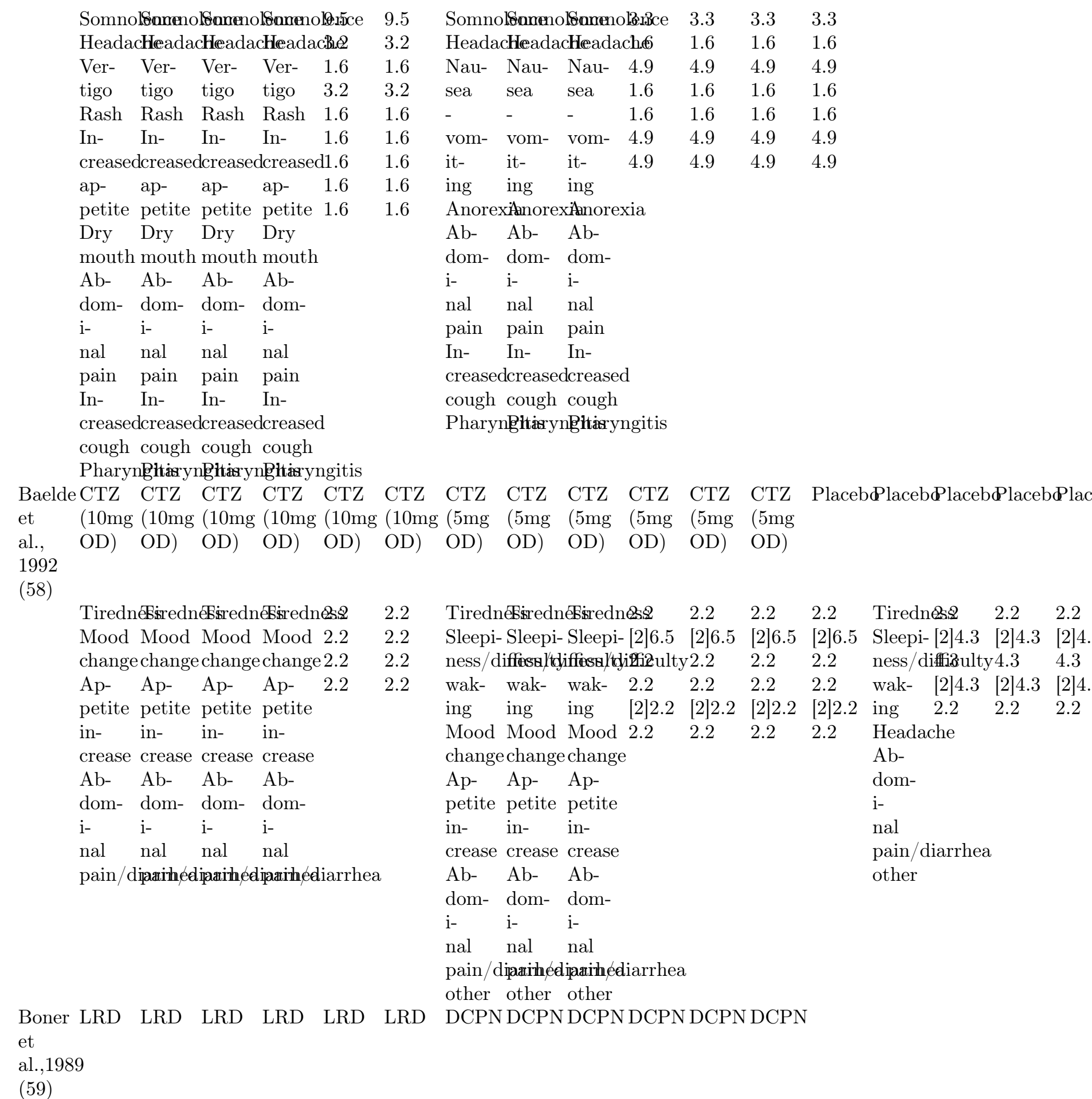




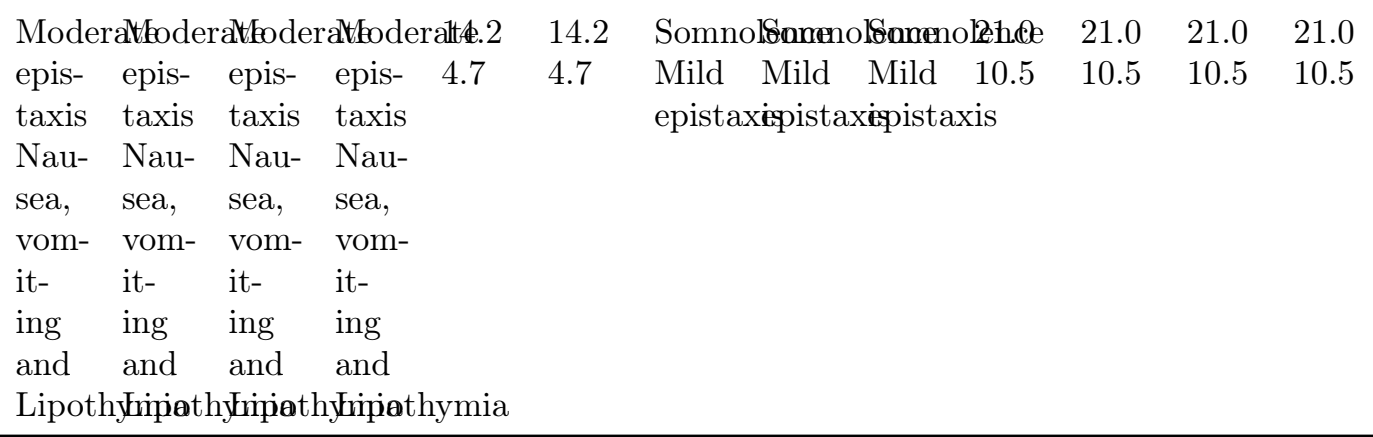

Abbreviations: $\mathrm{AE}=$ Adverse events; $\mathrm{BD}=$ bis in die (twice daily); $\mathrm{BLN}=$ Bilastine; $\mathrm{CTZ}=$ Cetirizine; $\mathrm{CPN}=$ Clorpheniramine CPHD $=$ Cyproheptadine $;$ DLRD $=$ Desloratadine $;$ DCPN $=$ Dexchlorpheniramine; FXD = Fexofenadine; KTF $=$ Ketotifen HCL; LRD = Loratadine; LCTZ = Levocetirizine; MLK = Montelukast; $\mathrm{ND}=$ No data; $\mathrm{OD}=$ omne in die (once daily); OXD = Oxotamide; PSD = Pseudoephedrine; $\mathrm{RPD}=$ Rupatadine; URTI $=$ Upper respiratory tract infection.

Figure Legends

Figure 1. Summary of evidence search and selection

* In total, 47 citations were included; there were two RCTs with data reported in two separate publications each.

Appendix

\section{Ovid MEDLINE and CENTRAL search strategy}

(Histamine H1 Antagonists.sh OR antihistamin*.af OR Cetirizine.af OR Loratadine.af OR Fexofenadine.af OR Levocetirizine.af OR Desloratadine.af OR Rupatadine.af OR Bilastine.af) AND (child*.tw OR infant*.tw OR toddler*.tw OR newborn*.tw OR neonate*.tw OR pediatric*.tw OR paediatric*.tw) 


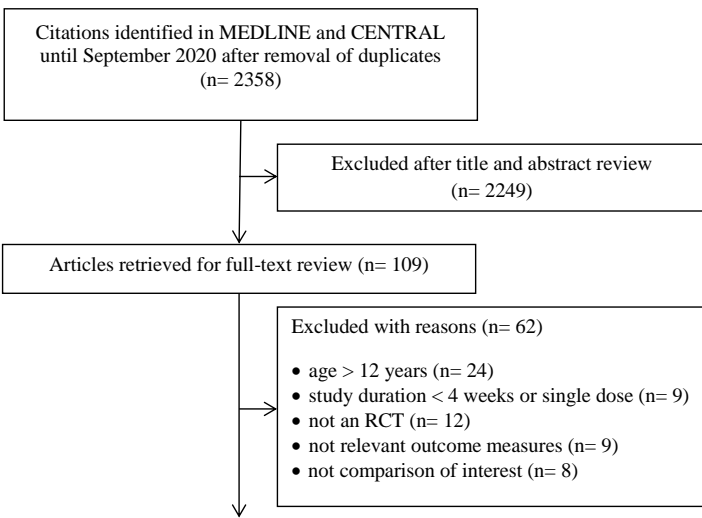

RCTs included in the systematic review $(\mathrm{n}=45)^{*}$ 